\title{
Horizontal branch morphology: A new photometric parametrization
}

\author{
M. Torelli ${ }^{1,2}$, G. Iannicola ${ }^{2}$, P. B. Stetson ${ }^{3}$, I. Ferraro ${ }^{2}$, G. Bono ${ }^{1,2}$, M. Salaris ${ }^{4}$, M. Castellani ${ }^{2}$, M. Dall'Ora ${ }^{5}$, \\ A. Fontana ${ }^{2}$, M. Monelli ${ }^{6,7}$, and A. Pietrinferni ${ }^{8}$ \\ 1 Department of Physics, Università di Roma Tor Vergata, Via della Ricerca Scientifica 1, 00133 Roma, Italy \\ 2 INAF-Osservatorio Astronomico di Roma, Via Frascati 33, 00078 Monte Porzio Catone, Italy \\ e-mail: marianna.torelli@inaf.it \\ 3 NRC-Herzberg, Dominion Astrophysical Observatory, 5071 West Saanich Road, Victoria, BC V9E 2E7, Canada \\ ${ }^{4}$ Astrophysics Research Institute, Liverpool John Moores University, IC2 Building, Liverpool Science Park, 146 Brownlow Hill, \\ Liverpool L3 5RF, UK \\ 5 INAF-Osservatorio Astronomico di Capodimonte, Salita Moiariello 16, 80131 Napoli, Italy \\ ${ }^{6}$ IAC - Instituto de Astrofisica de Canarias, Calle Via Lactea s/n, 38205 La Laguna, Spain \\ 7 Departamento de Astrofisica, Universidad de La Laguna, Avenida Astrofísico Francisco Sánchez s/n, 38200 Tenerife, Spain \\ 8 INAF - Osservatorio Astronomico d'Abruzzo, Via Mentore Maggini snc, Loc. Collurania, 64100 Teramo, Italy
}

Received 31 May 2019 / Accepted 19 July 2019

\begin{abstract}
Context. Theory and observations indicate that the distribution of stars along the horizontal branch of Galactic globular clusters mainly depends on the metal content. However, the existence of globular clusters with similar metal content and absolute age but different horizontal branch morphologies, suggests the presence of another parameter affecting the star distribution along the branch. Aims. To investigate the variation of the horizontal branch morphology in Galactic globular clusters, we define a new photometric horizontal branch morphology index, overcoming some of the limitations and degeneracies affecting similar indices available in the literature.

Methods. We took advantage of a sample of 64 Galactic globular clusters, with both space-based imaging data (Advanced Camera for Surveys survey of Galactic globular clusters) and homogeneous ground-based photometric catalogues in five different bands $(U, B$, $V, R, I)$. The new index, $\tau_{\mathrm{HB}}$, is defined as the ratio between the areas subtended by the cumulative number distribution in magnitude $(I)$ and in colour $(V-I)$ of all stars along the horizontal branch.

Results. This new index shows a linear trend over the entire range in metallicity $(-2.35 \leq[\mathrm{Fe} / \mathrm{H}] \leq-0.12)$ covered by our Galactic globular cluster sample. We found a linear relation between $\tau_{\mathrm{HB}}$ and absolute cluster ages. We also found a quadratic anti-correlation with $[\mathrm{Fe} / \mathrm{H}]$, becoming linear when we eliminate the age effect on $\tau_{\mathrm{HB}}$ values. Moreover, we identified a subsample of eight clusters that are peculiar according to their $\tau_{\mathrm{HB}}$ values. These clusters have bluer horizontal branch morphology when compared to typical ones of similar metallicity. These findings allow us to define them as the 'second parameter' clusters in the sample. A comparison with synthetic horizontal branch models suggests that they cannot be entirely explained with a spread in helium content.
\end{abstract}

Key words. stars: horizontal-branch - globular clusters: general

\section{Introduction}

Globular clusters are stellar systems that play a fundamental role in constraining the formation and evolution of galaxies (Searle \& Zinn 1978; VandenBerg et al. 2013; Leaman et al. 2013) and cosmological parameters. Dating back more than half a century (Sandage 1953), the absolute ages of Galactic globular clusters (GGCs) have been used to provide a lower limit to the age of the Universe (see e.g. Salaris \& Weiss 2002; Dotter et al. 2011; Monelli et al. 2015; Richer et al. 2013, and references therein) and estimate of the primordial helium abundance (see e.g. Zoccali et al. 2000; Salaris \& Cassisi 2005; Villanova et al. 2012). GGCs are also laboratories to investigate evolutionary (Chaboyer et al. 2017; Dotter et al. 2007; Brown et al. 2010; Weiss et al. 2004) and pulsational (Bono \& Stellingwerf 1994; Bono et al. 1999; Marconi et al. 2015) properties of old, lowmass stars. In this context, advanced evolutionary phases (red giant [RGs] and horizontal branch [HB] stars) have several advantages when compared with main sequence (MS) stars. They are a few magnitudes brighter and within the reach of spectroscopic investigations at the $8-10 \mathrm{~m}$ class telescopes. This means that they can be used for investigating the cluster dynamical evolution and interaction with the Galactic potential (Pancino et al. 2007; Zocchi et al. 2017; Calamida et al. 2017; Lanzoni et al. 2018). Moreover, their chemical abundances (iron peak, $\alpha$ , neutron capture elements) can be measured with high accuracy both in the optical (Carretta et al. 2014) and in the near-infrared (NIR) regime (D'Orazi et al. 2018).

Despite a general consistency between theory and observations concerning hydrogen and helium burning phases, we continue to face a number of long-standing open questions. Amongst them the morphology of the HB plays a pivotal role. Stars along the HB are low-mass $\left(M \approx 0.50-0.80 M_{\odot}\right)$, core-helium-burning stars and their distribution along the $\mathrm{HB}$ depends, at fixed initial chemical composition, on their envelope mass. Indeed, the envelope mass when moving from the red HB (RHB) to the extreme HB (EHB) decreases from $M_{\text {env }} \approx$ $0.30 M_{\odot}$ to $M_{\text {env }} \approx 0.0001 M_{\odot}$, while the effective temperature increases from $\approx 5500 \mathrm{~K}$ to $\approx 30000 \mathrm{~K}$.

The current empirical and theoretical evidence indicates that the HB morphology is affected by the initial metal content. 
Metal-poor clusters are mainly characterized by a blue HB morphology. This means that in these clusters HB stars are mainly distributed along the blue, hot, and extremely hot region. Metal-rich GGCs are generally characterized by a red HB morphology, that is, HB stars in these clusters are mainly distributed in the red (cool) region.

Although this theoretical and empirical framework appears well established, there is solid evidence that GGCs with similar chemical compositions display different HB morphologies. This suggests that the HB morphology was affected by at least a "second parameter" (see e.g. Sandage \& Wildey 1967, and references therein). This problem was defined as the "second parameter problem" and the clusters affected by this problem were called second parameter clusters.

During the last half-century several working hypotheses have been suggested to explain the second parameter problem. They include variations of the initial helium content (van den Bergh 1967; Sandage \& Wildey 1967), dynamical effects related to the cluster mass (e.g. Recio-Blanco et al. 2006), the cluster age (e.g. Searle \& Zinn 1978), dynamical evolution (e.g. Iannicola et al. 2009), or a combination of two or more of these - for example age and/or metallicity plus helium content, as suggested by Freeman \& Norris (1981), Gratton et al. (2010).

To quantify the extent of this second parameter problem, Lee (1989), Lee et al. (1994) suggested an HB morphology index based on star counts along the HB. It is defined as the difference between the number of stars that are bluer (B) and redder (R) than the RR Lyrae (RRL) instability strip, divided by the sum of the number of blue, red, and variable $(\mathrm{V})$ stars: $\mathrm{HBR}=\frac{B-R}{B+R+V}$. This HB morphology index has been quite popular, because it can be easily estimated from the theoretical and the observational point of view (star counts). However, it is prone to intrinsic degeneracies in both the metal-poor and the metal-rich regimes, in the sense that when the observed HB is bluer or redder than the RRL strip, HBR stays constant, irrespectively of the exact distribution of stars along the HB.

Similar HB morphology indices have been suggested in the literature, but using different cuts in colour. In particular, Buonanno (1993) suggested splitting HB stars bluer than the RRL instability strip into two blocks: stars hotter than the RRL instability strip and cooler than $(B-V)=-0.02$ were called $\mathrm{B} 1$, while those hotter than $(B-V)=-0.02$ were called B2. The new index had the key advantage of removing the degeneracy of the HBR index in the metal-poor regime, but it was still affected by degeneracies in the metal-rich regime.

Considering that almost all GGCs show the presence of multiple populations, Milone et al. (2014) introduced two new indices for describing the HB morphology. L1 is the difference in colour between the red giant branch (RGB) and the coolest red point of the $\mathrm{HB}$, while L2 is the colour extension of the HB. These two indices allowed the authors to identify three different GGC groups from the $\mathrm{L} 1-[\mathrm{Fe} / \mathrm{H}]$ diagram, and to find correlations of L1 with cluster age and metallicity. In addition, they found a variation of L2 with cluster luminosity (mass) and with helium content. This latter correlation is connected with the presence of multiple populations in globular clusters and it could be an additional ingredient to explain the HB colour extension. These two parameters are interesting, because they are correlated with the physics characterizing the HB stars. However, their definition seems to be very sensitive to the choice of the key points selected in colour magnitude diagrams (HB luminosity level, RGB).

In this work, we introduce a new HB morphology index based on the ratio between the areas subtended by the cumula- tive number distribution (CND) of star counts along the observed cluster $\mathrm{HB}$ in magnitude $\left(I\right.$-band) and in colour $(V-I): \tau_{\mathrm{HB}}=$ $A_{\mathrm{CND}}(I) / A_{\mathrm{CND}}(V-I)$. This new index has been calculated in a large sample (64) of GGCs, for which both space- and ground-based optical photometric catalogues are available. For the same sample of GGCs, we have also estimated the classical HBR index and performed a detailed comparison with $\tau_{\mathrm{HB}}$. We present evidence that our new index, in contrast with similar indices available in the literature, shows a well-defined correlation with cluster age and an anti-correlation with cluster iron abundance.

The structure of the paper is the following. We present in Sect. 2 our sample of GGCs with their photometric coverage. We also describe the method adopted for the separation between candidate cluster and field stars based on the comparison of star spectral energy distributions (SEDs). Section 3 deals with the classical HB morphology index HBR. We analyse its pros and cons and calculate its values for the entire cluster sample, together with its dependence on age and metallicity, while in Sect. 4 we do the same analysis for the L1 and L2 indices. In Sect. 5 we introduce our new HB morphology index, $\tau_{\mathrm{HB}}$, calculate its values for our GGC sample, and discuss pros and cons compared to HBR. In Sect. 6 we analyse and compare the relative difference between the classical HB morphology index and $\tau_{\mathrm{HB}}$ estimates when considering just space- or just ground-based observations. The correlation of our new index with the absolute age and the metallicity of the individual clusters is addressed in Sect. 7. Here we also identify the second parameter clusters in our sample that show very different estimates in $\tau_{\mathrm{HB}}$ compared to the ones attained by globulars with similar $[\mathrm{Fe} / \mathrm{H}]$ values. In Sect. 8 we compare $\tau_{\mathrm{HB}}$ with synthetic HB models specifically computed for this work. The summary of the results and a brief discussion concerning future developments of the project are outlined in Sect. 9. In Appendix A we list specific information on individual GGCs for which the photometric properties are uncertain either due to the lack of ground-based or space-based data, because the photometry does not cover the entire cluster area (tidal radius), or because of the small number of HB stars. In Appendix B we also included a few notes for the GGCs we defined as "outliers" in the analysis of the HB morphology.

\section{Globular cluster sample}

In this work we use a sample of 64 GGCs for which optical images are available from both space-based (Advanced Camera for Surveys (ACS)/Wide Field Channel (WFC) on board the Hubble Space Telescope (HST)) and ground-based observations. The relevant parameters for our cluster sample are listed in Table 1.

We took advantage of the photometric catalogues provided by Sarajedini et al. (2007), Dotter et al. (2011) in the context of the Hubble Space Telescope Treasury project, An ACS Survey of Galactic Globular Clusters ${ }^{1}$. This survey is based on a single ACS pointing across the centre of each cluster, observed through two complete orbits, one for the $F 606 W(\sim V)$ and one for the $F 814 W(\sim I)$. Cluster NGC 6715 represents an exception since it was observed for two orbits in each filter (Anderson et al. 2008). Thanks to these data we can avoid the crowding problems in the central regions of the clusters (the red region in Fig. 1 shows the field coverage for one of the globulars in the sample,

\footnotetext{
1 Photometry available at https://www.astro.ufl.edu/ ata/ public_hstgc/
} 
Table 1. Globular Clusters in the sample with parameters used in this work.

\begin{tabular}{|c|c|c|c|c|c|c|c|c|c|c|c|c|c|c|c|c|}
\hline ID & Name & RA (J 2000) ${ }^{(a)}$ & $\operatorname{Dec}(\mathrm{J} 2000)^{(a)}$ & $U$ & $B$ & $V$ & $R$ & I & $F 606 \mathrm{~W}$ & $F 814 W$ & $r_{t}{ }^{\prime()^{(a)}}$ & $\operatorname{HBR}^{(a)}$ & $\operatorname{Age}(\mathrm{Gyr})^{(b)}$ & $E(B-V)^{(c)}$ & {$[\mathrm{Fe} / \mathrm{H}]^{(d)}$} & $\mu^{(b)}$ \\
\hline NGC 0104 & 47 Tuc & $00: 24: 05.67$ & $-72: 04: 52.6$ & $\checkmark$ & $\checkmark$ & $\checkmark$ & $\checkmark$ & $\checkmark$ & $\checkmark$ & $\checkmark$ & 42.86 & -0.99 & $11.75 \pm 0.25$ & 0.03 & $-0.76 \pm 0.02$ & 13.25 \\
\hline NGC 0288 & & $00: 52: 45.24$ & $-26: 34: 57.4$ & $\checkmark$ & $\checkmark$ & $\checkmark$ & $\checkmark$ & $\checkmark$ & $\checkmark$ & $\checkmark$ & 12.94 & 0.98 & $11.50 \pm 0.38$ & 0.01 & $-1.32 \pm 0.02$ & 14.87 \\
\hline NGC 0362 & & 01:03:14.26 & $-70: 50: 55.6$ & $\checkmark$ & $\checkmark$ & $\checkmark$ & $\checkmark$ & $\checkmark$ & $\checkmark$ & $\checkmark$ & 16.11 & -0.87 & $10.75 \pm 0.25$ & 0.03 & $-1.30 \pm 0.04$ & 14.70 \\
\hline NGC 1261 & & 03:12:16.21 & $-55: 12: 58.4$ & $\checkmark$ & $\checkmark$ & $\checkmark$ & $\checkmark$ & $\checkmark$ & $\checkmark$ & $\checkmark$ & 7.28 & -0.71 & $10.75 \pm 0.25$ & 0.01 & $-1.27 \pm 0.08$ & 16.02 \\
\hline NGC 1851 & & 05:14:06.76 & $-40: 02: 47.6$ & $\checkmark$ & $\checkmark$ & $\checkmark$ & $\checkmark$ & $\checkmark$ & $\checkmark$ & $\checkmark$ & 11.7 & -0.36 & $11.00 \pm 0.38$ & 0.04 & $-1.18 \pm 0.08$ & 15.31 \\
\hline NGC 2298 & & $06: 48: 59.41$ & $-36: 00: 19.1$ & $\checkmark$ & $\checkmark$ & $\checkmark$ & $\checkmark$ & $\checkmark$ & $\checkmark$ & $\checkmark$ & 6.48 & 0.93 & - & 0.22 & $-1.96 \pm 0.04$ & - \\
\hline NGC 3201 & & $10: 17: 36.82$ & $-46: 24: 44.9$ & $\checkmark$ & $\checkmark$ & $\checkmark$ & $\checkmark$ & $\checkmark$ & $\checkmark$ & $\checkmark$ & 28.45 & 0.08 & $11.50 \pm 0.38$ & 0.26 & $-1.51 \pm 0.02$ & 13.29 \\
\hline NGC 4147 & & 12:10:06.30 & $+18: 32: 33.5$ & $\checkmark$ & $\checkmark$ & $\checkmark$ & $\checkmark$ & $\checkmark$ & $\checkmark$ & $\checkmark$ & 6.31 & 0.55 & $12.25 \pm 0.25$ & 0.03 & $-1.78 \pm 0.08$ & 16.33 \\
\hline NGC 4590 & M 68 & $12: 39: 27.98$ & $-26: 44: 38.6$ & $\checkmark$ & $\checkmark$ & $\checkmark$ & $\checkmark$ & $\checkmark$ & $\checkmark$ & $\checkmark$ & 30.34 & 0.17 & $12.00 \pm 0.25$ & 0.06 & $-2.27 \pm 0.04$ & 16.05 \\
\hline NGC 4833 & & 12:59:33.92 & $-70: 52: 35.4$ & $\checkmark$ & $\checkmark$ & $\checkmark$ & $\checkmark$ & $\checkmark$ & $\checkmark$ & $\checkmark$ & 17.85 & 0.93 & $12.50 \pm 0.50$ & 0.33 & $-1.89 \pm 0.05$ & - \\
\hline NGC 5024 & M53 & $13: 12: 55.25$ & $+18: 10: 05.4$ & $\checkmark$ & $\checkmark$ & $\checkmark$ & $\checkmark$ & $\checkmark$ & $\checkmark$ & $\checkmark$ & & 0.81 & $12.25 \pm 0.25$ & .03 & $-2.06 \pm 0.09$ & 16.31 \\
\hline NGC 5053 & & $13: 16: 2$ & $+17: 42: 00$ & $\checkmark$ & $\checkmark$ & $\checkmark$ & $\checkmark$ & $\checkmark$ & $\checkmark$ & $\checkmark$ & & & $12.25 \pm 0.38$ & 0.02 & $-2.30 \pm 0.08$ & 16.19 \\
\hline NGC 5272 & M3 & $13: 42: 11$ & $+28: 22$ & $\checkmark$ & $\checkmark$ & $\checkmark$ & $\checkmark$ & $\checkmark$ & $\checkmark$ & $\checkmark$ & 38.19 & & $11.75 \pm 0.25$ & & $-1.50 \pm 0.05$ & 14.99 \\
\hline NGC 5286 & & $13: 46: 2$ & $-51: 22: 27.3$ & $\checkmark$ & $\checkmark$ & $\checkmark$ & $\checkmark$ & $\checkmark$ & $\checkmark$ & $\checkmark$ & 8.36 & 0.80 & $12.25 \pm 0.38$ & 0.29 & $-1.70 \pm 0.07$ & 15.04 \\
\hline NGC 5466 & & $14: 05: 2$ & $+28: 32: 04.0$ & $\checkmark$ & $\checkmark$ & $\checkmark$ & $\checkmark$ & $\checkmark$ & $\checkmark$ & $\checkmark$ & 34.24 & 0.58 & $12.50 \pm 0.25$ & 0.02 & $-2.31 \pm 0.09$ & 16.09 \\
\hline NGC 5904 & M5 & $15: 18: 33.22$ & $+02: 0$ & $\checkmark$ & $\checkmark$ & $\checkmark$ & $\checkmark$ & $\checkmark$ & $\checkmark$ & $\checkmark$ & 28.4 & 0.31 & $11.50 \pm 0.25$ & 0.04 & $-1.33 \pm 0.02$ & 14.26 \\
\hline NGC 5927 & & $15: 28: 0$ & $-50: 4$ & $\checkmark$ & $\checkmark$ & $\checkmark$ & $\checkmark$ & $\checkmark$ & $\checkmark$ & $\checkmark$ & 16.68 & -1.00 & $10.75 \pm$ & 0.51 & $-0.29 \pm 0.07$ & 14.20 \\
\hline NGC 5986 & & $15: 46: 03$ & $-37: 4$ & $\checkmark$ & $\checkmark$ & $\checkmark$ & $\checkmark$ & $\checkmark$ & $\checkmark$ & $\checkmark$ & .52 & 0.97 & $12.25 \pm 0.75$ & 0.34 & $-1.63 \pm 0.08$ & - \\
\hline NGC 6093 & M 80 & $16: 17$ & $-22: 5$ & $\checkmark$ & $\checkmark$ & $\checkmark$ & $\checkmark$ & $\checkmark$ & $\checkmark$ & $\checkmark$ & & 0.93 & - & & & - \\
\hline NGC 6101 & & $16: 25: 4$ & $-72: 12:$ & $\checkmark$ & $\checkmark$ & $\checkmark$ & $\checkmark$ & $\checkmark$ & $\checkmark$ & $\checkmark$ & 7. & 0.84 & $12.25 \pm 0.50$ & 0 & $-1.98 \pm 0.07$ & - \\
\hline NGC 6121 & M4 & $16: 23: 35$ & $-26: 31:$ & $\checkmark$ & $\checkmark$ & $\checkmark$ & $\checkmark$ & $\checkmark$ & $\checkmark$ & $\checkmark$ & & -0.06 & & & & 11.07 \\
\hline NGC 6144 & & $16: 27: 13.86$ & $-26: 01: 24.6$ & & $\checkmark$ & $\checkmark$ & $\checkmark$ & $\checkmark$ & $\checkmark$ & $\checkmark$ & 25 & 1.00 & & & & - \\
\hline NGC 6171 & M 107 & 16:32: & & $\checkmark$ & $\checkmark$ & $\checkmark$ & $\checkmark$ & $\checkmark$ & $\checkmark$ & $\checkmark$ & & -0.73 & & & & 13.43 \\
\hline NGC 6205 & M 13 & $16: 41$ & $+36: 2$ & $\checkmark$ & $\checkmark$ & $\checkmark$ & $\checkmark$ & $\checkmark$ & $\checkmark$ & $\checkmark$ & 25.18 & 0.97 & & & & 14.39 \\
\hline NGC 6218 & M 12 & $16: 47: 14.18$ & $-01: 5$ & $\checkmark$ & $\checkmark$ & $\checkmark$ & $\checkmark$ & $\checkmark$ & $\checkmark$ & $\checkmark$ & 17.6 & 0.97 & $13.00 \pm 0.50$ & .17 & $-1.33 \pm 0.02$ & 13.52 \\
\hline NGC 6254 & M 10 & $16: 5$ & & $\checkmark$ & $\checkmark$ & $\checkmark$ & $\checkmark$ & $\checkmark$ & $\checkmark$ & $\checkmark$ & & 1.00 & & & \pm 0.02 & - \\
\hline NGC 6304 & & $17: 1$ & $-29: 2$ & & & & & & $\checkmark$ & $\checkmark$ & 25 & -1.00 & & .52 & 0.07 & 13.81 \\
\hline NGC 6341 & M 92 & $17: 17$ & $+43: 08$ & $\checkmark$ & $\checkmark$ & $\checkmark$ & $\checkmark$ & $\checkmark$ & $\checkmark$ & $\checkmark$ & & 0.91 & & & 0.05 & 14.66 \\
\hline NGC 6352 & & 17:26: & $-48: 25: 1$ & & $\checkmark$ & $\checkmark$ & & $\checkmark$ & $\checkmark$ & $\checkmark$ & 10.51 & -1.00 & \pm 0.38 & & $=0.05$ & 13.36 \\
\hline NGC 6362 & & & & & $\checkmark$ & $\checkmark$ & $\checkmark$ & $\checkmark$ & $\checkmark$ & $\checkmark$ & 16.67 & -0.58 & & & & 14.36 \\
\hline NGC 6366 & & & & $\checkmark$ & $\checkmark$ & $\checkmark$ & $\checkmark$ & $\checkmark$ & $\checkmark$ & $\checkmark$ & 15.2 & -0.97 & $0 \pm 0$ & & & 12.40 \\
\hline NGC 6388 & & & & & $\checkmark$ & $\checkmark$ & & $\checkmark$ & $\checkmark$ & $\checkmark$ & & - & - & & \pm 0.04 & - \\
\hline NGC 6397 & & & $-53: 4$ & $\checkmark$ & $\checkmark$ & $\checkmark$ & $\checkmark$ & $\checkmark$ & $\checkmark$ & $\checkmark$ & 15.81 & 1.00 & $13.00 \pm 0.25$ & & $-1.99 \pm 0.02$ & - \\
\hline NGC 6426 & & & $+03: 10$ & & & & & & $\checkmark$ & $\checkmark$ & 13.23 & 0.58 & - & 0 & -2.15 & - \\
\hline NGC 6441 & & & $-37: 0$ & $\checkmark$ & $\checkmark$ & $\checkmark$ & $\checkmark$ & $\checkmark$ & $\checkmark$ & $\checkmark$ & 8.0 & - & - & & $-0.44 \pm 0.07$ & - \\
\hline NGC 6496 & & & & $\checkmark$ & $\checkmark$ & $\checkmark$ & $\checkmark$ & $\checkmark$ & $\checkmark$ & $\checkmark$ & & -1.00 & $10.75 \pm 0.38$ & & & 14.93 \\
\hline NGC 6535 & & & & $\checkmark$ & $\checkmark$ & $\checkmark$ & & $\checkmark$ & $\checkmark$ & $\checkmark$ & & 1.00 & 50 & & \pm 0.07 & 13.88 \\
\hline & & & & $\checkmark$ & $\checkmark$ & $\checkmark$ & & $\checkmark$ & $\checkmark$ & $\checkmark$ & & & & & & - \\
\hline NGC 6584 & & & $-52: 12: 56.8$ & & $\checkmark$ & $\checkmark$ & $\checkmark$ & $\checkmark$ & $\checkmark$ & $\checkmark$ & 9.3 & -0.15 & & & 0.09 & 15.54 \\
\hline & & & & & & & & & $\checkmark$ & $\checkmark$ & 20.55 & -1.00 & & & & 14.47 \\
\hline NGC 6637 & M 69 & & & & $\checkmark$ & $\checkmark$ & & $\checkmark$ & $\checkmark$ & $\checkmark$ & 8.3 & -1. & & & $=0.07$ & 14.70 \\
\hline NGC 6652 & & & & & & $\checkmark$ & & $\checkmark$ & $\checkmark$ & $\checkmark$ & & -1.00 & & & 0.14 & 14.90 \\
\hline & M 22 & & & $\checkmark$ & $\checkmark$ & $\checkmark$ & $\checkmark$ & $\checkmark$ & $\checkmark$ & & & & & & & - \\
\hline NGC 6681 & M70 & & $-32: 17: 31.6$ & $\checkmark$ & $\checkmark$ & $\checkmark$ & $\checkmark$ & $\checkmark$ & $\checkmark$ & $\checkmark$ & 7.9 & & & & \pm 0.08 & 14.78 \\
\hline NGC 6717 & $\mathrm{Pal} 9$ & & $-22: 42: 05.3$ & & & $\checkmark$ & $\checkmark$ & $\checkmark$ & $\checkmark$ & $\checkmark$ & & & & & & 14.17 \\
\hline NGC 6723 & & 18: & $-36: 37: 56.1$ & & $\checkmark$ & $\checkmark$ & & $\checkmark$ & $\checkmark$ & $\checkmark$ & 10.51 & -0.08 & $.25 \pm 0.25$ & & $-1.10 \pm 0.07$ & 14.23 \\
\hline & & & & $\checkmark$ & $\checkmark$ & $\checkmark$ & $\checkmark$ & $\checkmark$ & $\checkmark$ & $\checkmark$ & & & & & & - \\
\hline 6779 & M 56 & & & $\checkmark$ & $\checkmark$ & $\checkmark$ & $\checkmark$ & $\checkmark$ & $\checkmark$ & $\checkmark$ & & & & & .09 & - \\
\hline & M 55 & & & $\checkmark$ & $\checkmark$ & $\checkmark$ & $\checkmark$ & $\checkmark$ & $\checkmark$ & $\checkmark$ & 16 & & & & & 13.62 \\
\hline 6838 & M 71 & & & $\checkmark$ & $\checkmark$ & $\checkmark$ & $\checkmark$ & $\checkmark$ & $\checkmark$ & $\checkmark$ & 8. & -1.00 & & & & 12.67 \\
\hline NGC 6934 & & $20: 34$ & & $\checkmark$ & $\checkmark$ & $\checkmark$ & $\checkmark$ & $\checkmark$ & $\checkmark$ & $\checkmark$ & & & & & \pm 0.09 & 16.05 \\
\hline & M72 & & & $\checkmark$ & $\checkmark$ & $\checkmark$ & $\checkmark$ & $\checkmark$ & $\checkmark$ & $\checkmark$ & & & $.50 \pm 0.25$ & 0.0 & & 16.05 \\
\hline NGC 7006 & & & & $\checkmark$ & $\checkmark$ & $\checkmark$ & $\checkmark$ & $\checkmark$ & $\checkmark$ & $\checkmark$ & & -0.28 & - & & $5 \pm 0.06$ & - \\
\hline & M 15 & & & $\checkmark$ & $\checkmark$ & $\checkmark$ & $\checkmark$ & 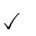 & $\checkmark$ & & & & & & & \\
\hline NGC 7089 & M2 & $21: 33: 27.02$ & $-00: 49: 23.7$ & $\checkmark$ & $\checkmark$ & $\checkmark$ & $\checkmark$ & $\checkmark$ & $\checkmark$ & $\checkmark$ & 21. & & $.50 \pm 0.25$ & 0.04 & $-1.66 \pm 0.07$ & 15.41 \\
\hline NGC 7099 & M 30 & $21: 40: 22.12$ & & $\checkmark$ & $\checkmark$ & $\checkmark$ & $\checkmark$ & $\checkmark$ & $\checkmark$ & $\checkmark$ & & 0.89 & & 0.0 & $-2.33 \pm 0.02$ & 14.60 \\
\hline & & & & $\checkmark$ & $\checkmark$ & $\checkmark$ & & $\checkmark$ & $\checkmark$ & $\checkmark$ & & & $12.00 \pm 0.38$ & 0.11 & $-1.74 \pm 0.08$ & 17.25 \\
\hline IC 4499 & & & $-82: 12: 49.3$ & $\checkmark$ & $\checkmark$ & $\checkmark$ & $\checkmark$ & $\checkmark$ & $\checkmark$ & $\checkmark$ & 12.35 & & - & 0.22 & $-1.62 \pm 0.09$ & - \\
\hline Lynga 7 & & & & & & & & & $\checkmark$ & $\checkmark$ & - & -1.0 & - & 1.06 & - & - \\
\hline Pal 2 & & 04:46:05.91 & $+31: 22: 53.4$ & & $\checkmark$ & $\checkmark$ & $\checkmark$ & $\checkmark$ & $\checkmark$ & $\checkmark$ & 6.76 & - & - & 1.21 & $-1.29 \pm 0.09$ & - \\
\hline Rup 106 & & & & & $\checkmark$ & $\checkmark$ & $\checkmark$ & & $\checkmark$ & $\checkmark$ & 50 & & - & 0.17 & $-1.78 \pm 0.08$ & - \\
\hline & & :17:43.92 & $-34: 39: 27.8$ & & $\checkmark$ & $\checkmark$ & $\checkmark$ & $\checkmark$ & $\checkmark$ & $\checkmark$ & 7.27 & -1.00 & - & 0.09 & $-0.12 \pm 0.08$ & - \\
\hline Terzan 8 & & 19:41:44.41 & $-33: 59: 58.1$ & & & $\checkmark$ & $\checkmark$ & & $\checkmark$ & & 4.0 & 1.00 & $13.00 \pm 0.38$ & 0.15 & - & 17.14 \\
\hline
\end{tabular}

Notes. Columns are: J 2000 coordinates, data availability in ground-based and space-based bands, tidal radius in arcmin, HBR index, Age in Gyr, colour excess $E(B-V),[\mathrm{Fe} / \mathrm{H}]$, apparent visual distance modulus $\mu$.

References. ${ }^{(a)}$ Harris (1996); ${ }^{(b)}$ VandenBerg et al. (2013); ${ }^{(c)}$ Dutra \& Bica (2000); ${ }^{(d)}$ Carretta et al. (2009).

NGC 5053) and reach very faint magnitudes $(I \sim 26.0$, see ACS colour-magnitude diagram (CMD) in Fig. 2).

To cover a significant fraction of the body of each cluster we adopted the multi-band $(U, B, V, R, I)$ optical catalogues provided by one of the authors (PBS, see for example Stetson et al. 2005, 2014, 2019), based on images collected with several ground-based telescopes. The ground-based data allow us to reach the tidal radius for most of the globulars. The blue line in Fig. 1 shows the tidal radius of NGC 5053. There are a few clusters (see Appendix A) for which we cannot reach the tidal radius 


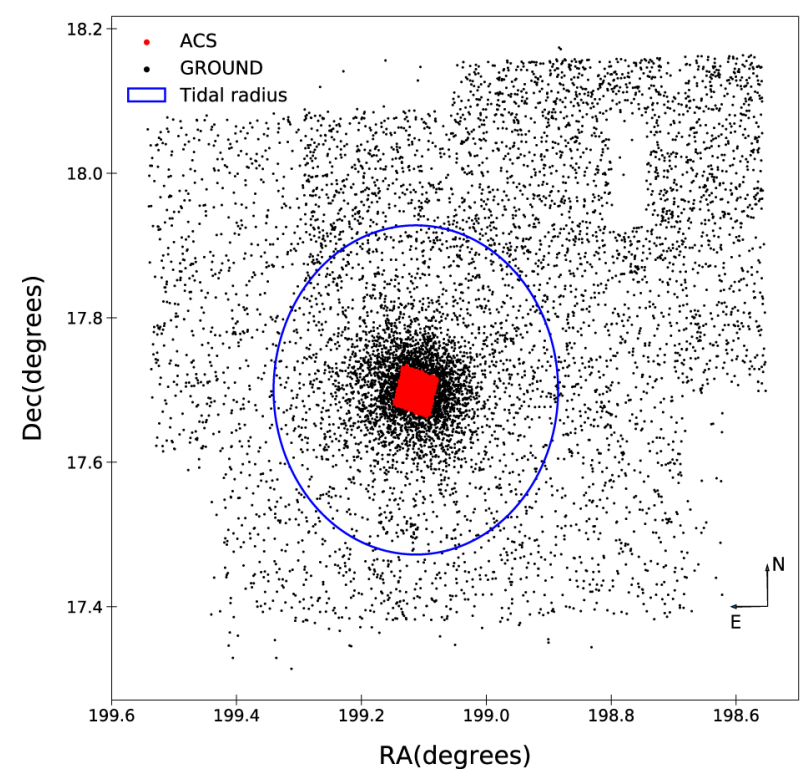

Fig. 1. Sky distribution of ground-based (black dots) and space-based (red dots) data for the cluster NGC 5053. North is up and east is to the left.

with our data while still covering the main body of the cluster. For 47 GGCs we have data in all available photometric bands from both space- and ground-based facilities.

Thanks to the fact that all the clusters in the sample have data in $B, V, I$ bands (except for NGC 6426, NGC 6624, NGC 6652, Lynga 7, and Palomar 2, see Appendix A), we based the cluster and field stars' separation on the SED of the stars in these bands, following the procedure described in Di Cecco et al. (2015), Calamida et al. (2017). We selected first the bona fide cluster stars considering only the central region, since we expect that it is less contaminated by field stars. Then we compared the SEDs of these stars to the ones in the total catalogue, to separate cluster stars from the field. We note that for stars with space data only we did not make any selection, because we expect negligible contamination by field stars in the ACS field of view (FoV). Therefore, in this case we took advantage of the entire ACS catalogues.

Moreover, when we had measurements in the $V$ and $I$ bands from both ACS and ground-based telescopes, we preferred the first ones for their higher signal-to-noise ratio $(\mathrm{S} / \mathrm{N})$ values. We used the ACS coverage for the central region of our globulars within the ACS FoV $\left(\sim 4^{\prime}\right)$, while outside up to the tidal radius we adopted the ground-based observations.

An example of our selection is shown in Fig. 3, which displays the $V, B-I$ CMD of NGC 5286 for total (left panel), cluster (central panel), and field (right panel) stars. We can appreciate from the figure that the joint catalogues allow us to cover the entire evolution of the stars in the globulars, from the faint part of the MS to the brighter region of the RGB-tip and asymptotic giant branch (AGB) in the CMD.

\section{The HBR morphology index}

As already discussed in Sect. 1, the traditional HB morphology index is defined as

$\mathrm{HBR}=\frac{B-R}{B+R+V}$,

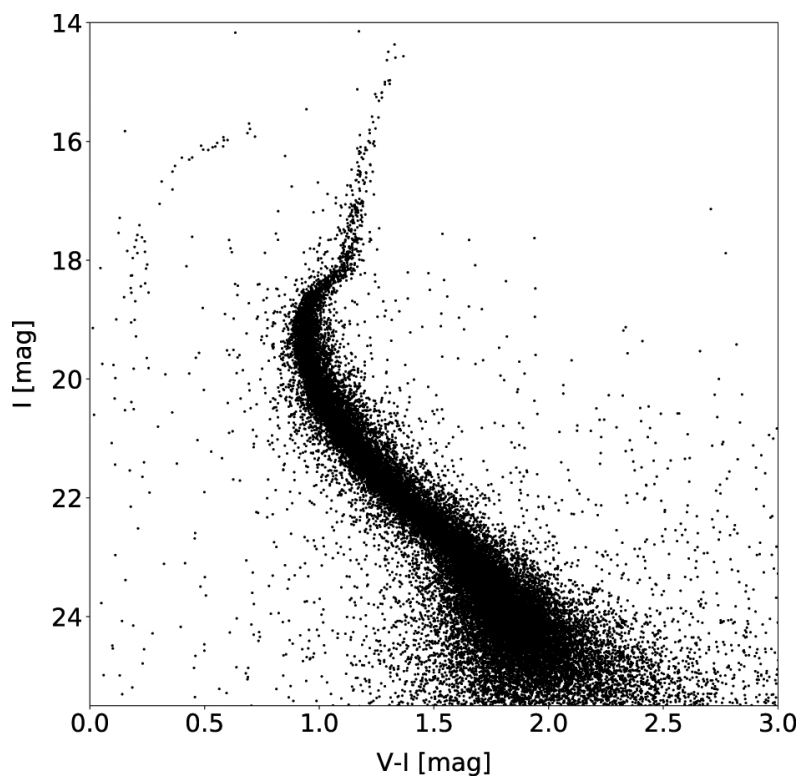

Fig. 2. Cluster NGC $5286 I, V-I$ CMD based only on ACS-HST data.
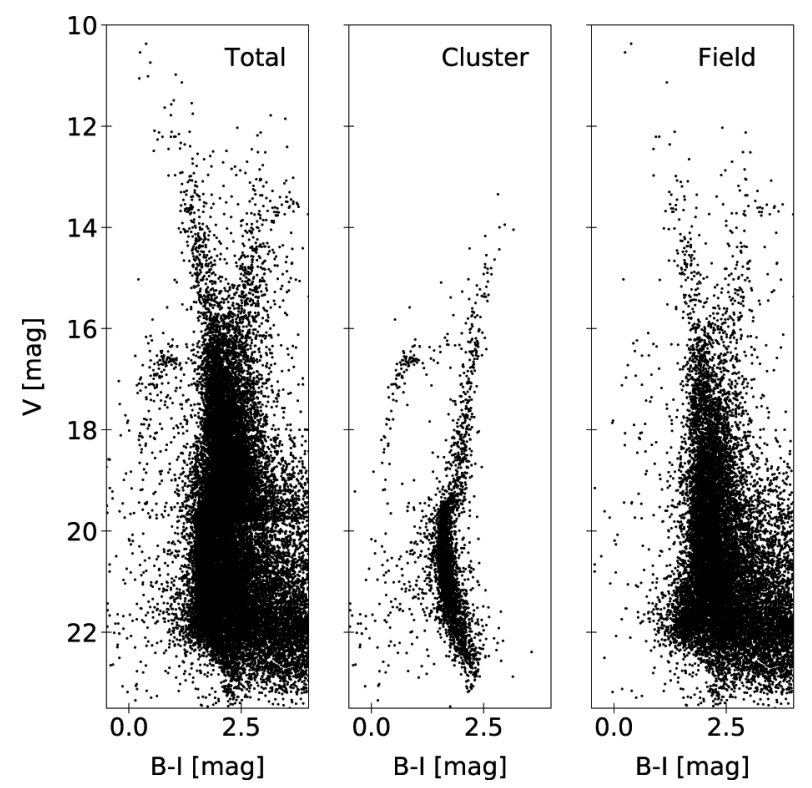

Fig. 3. Cluster NGC5286 V,B - I CMD. Left panel: all stars. Central panel: our candidate cluster stars. Right panel: candidate field stars. Candidate cluster and field stars have been selected following the method described in the main text.

where $B$ is the number of HB stars bluer than the blue (hot) edge of the RRL instability strip, $V$ is the number of RR Lyrae stars, and $R$ is the number of HB stars redder than the red (cold) edge of the RRL instability strip. This HB morphology index has several advantages when compared with other observables (HB luminosity level, colour distribution) connected with the helium burning phases.

First, when considered with the comparison between predicted and observed HB luminosity levels, HB star counts are independent of uncertainties affecting the cluster distance and are minimally affected by uncertainties in cluster reddening and the possible occurrence of differential reddening. Moreover, the comparison between the predicted and observed number of $\mathrm{HB}$ stars is less prone to uncertainties affecting the transformations of theoretical predictions into the observational plane. 
In the comparison between theory and observations, the HBR index accounts for the global distribution of stars along the HB. On the other hand, the comparison between the predicted and observed HB luminosity levels is mainly restricted to the RRL instability strip, that is, the truly horizontal region of the HB. However, a minor fraction of GGCs hosts a sizeable sample of RRLs to properly define the HB luminosity level.

One of the main cons of the HBR index is that it describes a global property of HB stars. This means that it does not trace the detailed stellar distribution along the HB. It is also affected by severe degeneracies both in the metal-poor and in the metal-rich regimes. This means that a significant fraction of GGCs attain values close either to 1 (blue $\mathrm{HB}$ morphology) or to -1 (red HB morphology), irrespective of the exact colour distribution of stars.

Despite the stated limitations we calculated values of the HBR index for our GGC sample. For each cluster we defined two boxes including candidate blue (B) and red (R) HB stars. The colour ranges for the boxes were fixed using either predicted or empirical edges for the RRL instability strip. Stars located in the RRL instability strip were neglected. To account for the number of variables we took advantage of the Clement catalogue of variable stars in GGCs. This catalogue was originally presented in Clement et al. (2001), but it is constantly updated ${ }^{2}$. For each cluster, it gives the number of variable stars together with their main pulsation parameters: pulsation period, mean magnitude and colour, and luminosity amplitude. For our analysis we took into account only confirmed cluster RRL stars, meaning that candidate cluster RRLs were not included.

Throughout our analysis, we adopted the homogeneous metallicity scale provided by Carretta et al. (2009). The authors estimated the iron abundances from high resolution spectra collected with the Fibre Large Srray Multi Element Spectrograph (FLAMES) at the Very Large Telescope (VLT), covering the entire metallicity range of GGCs. To increase the sample size they also re-calibrated the most common metallicity scales available in the literature (Zinn \& West 1984; Kraft \& Ivans 2003; Carretta \& Gratton 1997). For two clusters in our sample (NGC 6624 and Lynga 7) we adopted the metallicity estimate available in the 2010 version of the Harris (1996) catalogue ${ }^{3}$.

To validate the current estimates of the HBR index with similar estimates available in the literature, Fig. 4 shows the comparison of our values with those provided by Harris in the 2003 version of his catalogue (Harris 1996). Data plotted in this figure show that both sets agree well over the entire metallicity range. Indeed, the difference is on average smaller than $20 \%$. There are a few outliers (NGC 1851 and NGC 4590) for which the difference is approximately $30 \%-40 \%$, but they are affected by a higher field-star contamination than the others, despite our homogeneous cluster and field star separation.

For each GGC in our sample, Table 2 gives its name (Cols. 1 and 2), the metallicity (Col. 3), and the number of blue (B) and red (R) stars (Cols. 4 and 5). In Col. 6 the values of the HBR' index, defined as HBR $=\mathrm{HBR}+2$, are listed. The reason for this change is the following. From Fig. 4 we can see that the errors on the HBR index are small and not realistic. We define the uncertainty of the measured HBR as

$$
\sigma(\mathrm{HBR})=\frac{B-R}{B+R+V} \cdot\left(\frac{1}{\sqrt{B-R}}+\frac{1}{\sqrt{B+R+V}}\right),
$$

\footnotetext{
2 http://www . astro.utoronto.ca/ cclement/cat/listngc. html

3 http://physwww.mcmaster.ca/ harris/mwgc.dat
}

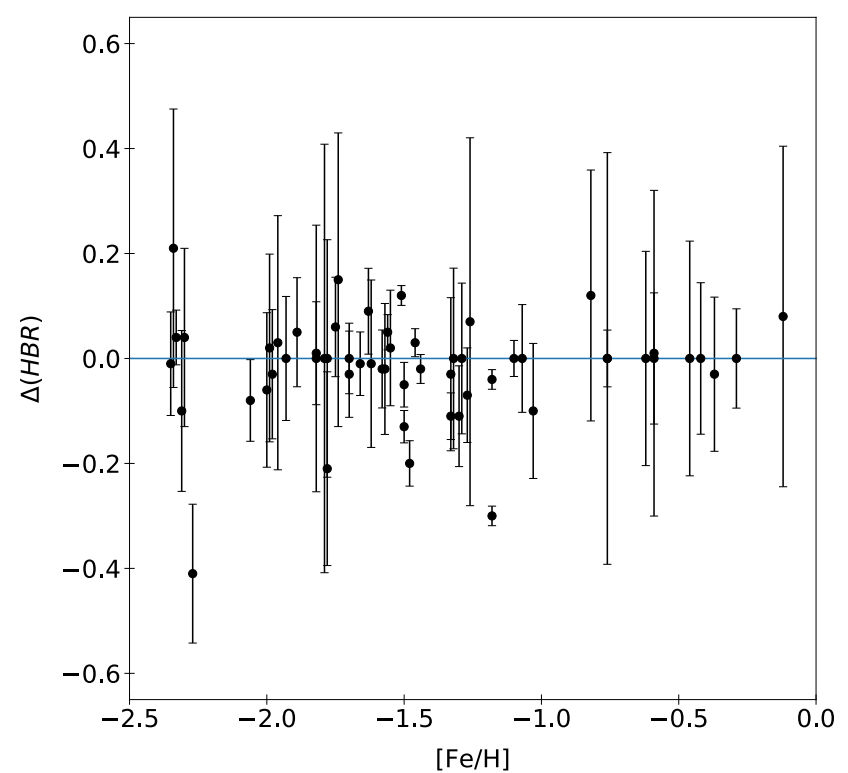

Fig. 4. Difference between Harris (1996) values (2003 version) for HBR and our measurements as a function of $[\mathrm{Fe} / \mathrm{H}]$.

which takes into account the Poisson uncertainties in the star counts. The errors provided by this formula vanish as soon as $B$ and $R$ attain similar values, that is when HBR approaches zero. This is an intrinsic limitation affecting the definition of the HBR index. To overcome this problem we decided to use $\mathrm{HBR}^{\prime}$.

To investigate the dependence of the HB morphology on intrinsic cluster parameters, Fig. 5 shows the $\mathrm{HBR}^{\prime}$ index as a function of iron content for the current sample. Data plotted in this figure display some well established correlations. The HB morphology becomes systematically bluer when moving from metal-poor to more metal-rich GGCs.

There is a well-defined degeneracy in the metal-intermediate regime $(-1.6 \leq[\mathrm{Fe} / \mathrm{H}] \leq-1)$ in which GGCs with very similar metal abundances (the typical error on cluster metallicity being on the order of 0.1 dex - see horizontal error bar in the left bottom corner) cover a broad range in $\mathrm{HBR}^{\prime}$ values. This means a variation from a very blue to a very red $\mathrm{HB}$ morphology, the well known second parameter problem. Figure 5 shows that $\mathrm{HBR}^{\prime}$ is affected by degeneracy in its extreme values, $\mathrm{HBR}^{\prime} \sim-1$ (metalrich regime) and $\mathrm{HBR}^{\prime} \sim+1$ (metal-poor regime). This means that $\mathrm{HBR}^{\prime}$ has a low dynamical range attaining similar values for clusters with different metallicity.

Cluster age has been considered together with iron content as one of the main culprits affecting the HB morphology. We took advantage of the homogeneous cluster age estimates provided by VandenBerg et al. (2013), Leaman et al. (2013) for the same GGCs, to investigate the correlation with $\mathrm{HBR}^{\prime}$ index. They estimated the cluster ages as follows. Firstly, they de-reddened the HB stars and then estimated the absolute visual magnitude by fitting a theoretical zero-age horizontal branch (ZAHB) to the lower bound of the HB star distributions. The absolute age of the globulars is estimated by the isochrone which, at fixed chemical composition, best matches the CMD from the main sequence turn off (MSTO) point to the beginning of the sub giant branch (SGB). Data plotted in Fig. 6 show that the HB morphology, at fixed cluster age, can move from very blue to very red. Indeed, the HBR' index, within the uncertainty on the cluster age ( $\sim 1 \mathrm{Gyr}$, displayed by the error bar in the left bottom corner), ranges from 1 to 3 . This evidence shows that the HBR' index 
A\&A 629, A53 (2019)

Table 2. Parameters and observational HB morphology indices for the GGCs in the sample.

\begin{tabular}{|c|c|c|c|c|c|c|c|c|c|c|c|}
\hline ID & Name & {$[\mathrm{Fe} / \mathrm{H}]$} & B & $\mathrm{R}$ & $\mathrm{HBR}^{\prime}$ & L1 ${ }^{(a)}$ & $\mathrm{L} 2^{(a)}$ & $\partial Y^{(b)}$ & $\mathrm{V}(\mathrm{HB})^{(c)}$ & $A_{\mathrm{CND}}(V-I)$ & $\tau_{\mathrm{HB}}$ \\
\hline NGC 0104 & 47 Tuc & -0.76 & 0 & 1336 & $1.01 \pm 0.04$ & $0.078 \pm 0.005$ & $0.068 \pm 0.006$ & $0.049 \pm 0.005$ & 14.06 & $137.9 \pm 11.7$ & $1.58 \pm 0.01$ \\
\hline NGC 0288 & & -1.32 & 130 & 0 & $2.98 \pm 0.12$ & $0.534 \pm 0.086$ & $0.337 \pm 0.086$ & $0.016 \pm 0.012$ & 15.44 & $47.8 \pm 6.9$ & $9.39 \pm 0.37$ \\
\hline NGC 0362 & & -1.30 & 12 & 282 & $1.24 \pm 0.07$ & $0.086 \pm 0.005$ & $0.608 \pm 0.064$ & $0.026 \pm 0.008$ & 15.44 & $126.1 \pm 11.2$ & $3.24 \pm 0.01$ \\
\hline NGC 1261 & & -1.27 & 35 & 201 & $1.36 \pm 0.06$ & $0.088 \pm 0.005$ & $0.644 \pm 0.038$ & $0.019 \pm 0.007$ & 16.70 & $114.0 \pm 10.7$ & $2.42 \pm 0.02$ \\
\hline NGC 1851 & & -1.18 & 137 & 156 & $1.95 \pm 0.01$ & $0.098 \pm 0.004$ & $0.679 \pm 0.010$ & $0.025 \pm 0.006$ & 16.09 & $90.9 \pm 9.5$ & $3.51 \pm 0.06$ \\
\hline NGC 2298 & & -1.96 & 53 & 1 & $2.90 \pm 0.17$ & $0.486 \pm 0.020$ & $0.267 \pm 0.023$ & $0.011 \pm 0.012$ & 16.11 & $57.7 \pm 7.6$ & $5.97 \pm 0.17$ \\
\hline NGC 3201 & & -1.51 & 90 & 103 & $1.96 \pm 0.01$ & $0.106 \pm 0.015$ & $0.649 \pm 0.022$ & $0.028 \pm 0.032$ & 14.76 & $88.7 \pm 9.4$ & $2.80 \pm 0.05$ \\
\hline NGC 4147 & & -1.78 & 61 & 2 & $2.76 \pm 0.13$ & $0.271 \pm 0.027$ & $0.476 \pm 0.029$ & - & 17.02 & $61.7 \pm 7.9$ & $5.96 \pm 0.12$ \\
\hline NGC 4590 & M 68 & -2.27 & 61 & 1 & $2.58 \pm 0.09$ & $0.205 \pm 0.029$ & $0.524 \pm 0.030$ & $0.012 \pm 0.009$ & 15.68 & $74.8 \pm 8.6$ & $4.43 \pm 0.07$ \\
\hline NGC 4833 & & -1.89 & 280 & 8 & $2.88 \pm 0.07$ & $0.287 \pm 0.037$ & $0.538 \pm 0.037$ & $0.051 \pm 0.009$ & 15.60 & $55.2 \pm 7.4$ & $6.21 \pm 0.28$ \\
\hline NGC 5024 & M 53 & -2.06 & 495 & 0 & $2.89 \pm 0.06$ & $0.158 \pm 0.035$ & $0.602 \pm 0.036$ & $0.04 \pm 0.008$ & & & $6.67 \pm 0.16$ \\
\hline NGC 5053 & & -2.30 & 34 & 8 & $2.46 \pm 0.12$ & $0.223 \pm 0.090$ & $0.439 \pm 0.090$ & $0.004 \pm 0.35$ & 16.69 & $74.2 \pm 8.6$ & $4.35 \pm 0.11$ \\
\hline NGC 5272 & M3 & -1.50 & 213 & 392 & $2.21 \pm 0.02$ & $0.150 \pm 0.016$ & $0.613 \pm 0.018$ & $0.041 \pm 0.009$ & 15.64 & $81.5 \pm 9.0$ & $4.13 \pm 0.05$ \\
\hline NGC 5286 & & -1.70 & 548 & 36 & $2.80 \pm 0.05$ & $0.213 \pm 0.034$ & $0.670 \pm 0.035$ & $0.044 \pm 0.004$ & 16.63 & $53.7 \pm 7.3$ & $6.17 \pm 0.30$ \\
\hline NGC 5466 & & -2.31 & 72 & 5 & $2.62 \pm 0.11$ & $0.225 \pm 0.062$ & $0.457 \pm 0.063$ & $0.007 \pm 0.024$ & & & $.02 \pm 0.10$ \\
\hline NGC 5904 & M5 & -1.33 & 98 & 155 & $2.42 \pm 0.03$ & $0.150 \pm 0.012$ & $0.681 \pm 0.014$ & $0.037 \pm 0.007$ & 15.07 & $68.9 \pm$ & $.04 \pm 0.14$ \\
\hline NGC 5927 & & -0.29 & 0 & 447 & $1.00 \pm 0.07$ & $0.043 \pm 0.003$ & $0.062 \pm 0.004$ & $0.055 \pm 0.015$ & & $135.2 \pm 11.6$ & $0.97 \pm 0.01$ \\
\hline NGC 5986 & & -1.63 & 459 & 26 & $2.88 \pm 0.06$ & $0.460 \pm 0.052$ & $0.443 \pm 0.053$ & $0.031 \pm 0$. & & & $7.85 \pm 0.46$ \\
\hline NGC 6093 & M 80 & -1.75 & 332 & 16 & $2.87 \pm 0.07$ & $0.464 \pm 0.059$ & & $0.027 \pm 0$. & & & $7.86 \pm 0.36$ \\
\hline NGC 6101 & & -1.98 & 169 & 17 & $2.87 \pm 0.09$ & $0.485 \pm 0.025$ & $0.223 \pm 0.025$ & $0.017 \pm 0.011$ & 16.60 & & $5.43 \pm 0.15$ \\
\hline NGC 6121 & M4 & -1.18 & 51 & 55 & $1.98 \pm 0.01$ & $0.120 \pm 0.020$ & $0.569 \pm 0.020$ & $0.014 \pm 0.006$ & & & \\
\hline NGC 6144 & & -1.82 & 62 & 0 & $3.00 \pm 0.18$ & $0.533 \pm 0.024$ & $0.229 \pm 0$. & $0.017 \pm 0$. & & & \\
\hline NGC 6171 & M 107 & -1.03 & 12 & 89 & $1.37 \pm 0.09$ & $0.100 \pm 0.014$ & $0.513 \pm 0.074$ & $0.024 \pm 0.0$ & & $116.2=$ & $0.28 \pm 0.03$ \\
\hline NGC 6205 & M 13 & -1.58 & 705 & 0 & $2.99 \pm 0.05$ & $0.527 \pm 0.013$ & $0.441 \pm 0.012$ & & & & $13.37 \pm 0.72$ \\
\hline NGC 6218 & M 12 & -1.33 & 188 & 0 & $3.00 \pm 0.10$ & $0.561 \pm 0.034$ & $0.299 \pm 0.035$ & $0.011 \pm 0.011$ & 14.60 & $49.8 \pm 7.1$ & $9.05 \pm 0.32$ \\
\hline NGC 6254 & M 10 & -1.57 & 257 & 0 & $3.00 \pm 0.09$ & 0.032 & $0.260 \pm 0.033$ & $0.029 \pm 0$. & & $40.6 \pm 6.4$ & $11.03 \pm 0.66$ \\
\hline NGC 6304 & & -0.37 & 0 & 170 & $1.03 \pm 0.10$ & $0.062 \pm 0.007$ & $0.060 \pm 0.004$ & $0.025 \pm 0.006$ & 15.60 & 0 & 0 \\
\hline NGC 6341 & M 92 & -2.25 & 340 & 6 & $2.92 \pm 0.07$ & $0.261 \pm 0.075$ & $0.542 \pm 0.075$ & $0.039 \pm 0.006$ & 15.10 & $47.4 \pm 6.9$ & $8.95 \pm 0.30$ \\
\hline NGC 6352 & & -0.62 & 0 & 96 & $1.00 \pm 0.14$ & 0.007 & 0.003 & $0.027 \pm$ & 5.13 & $130.6 \pm$ & $44 \pm 0.01$ \\
\hline NGC 6362 & & -1.07 & 56 & 135 & $1.65 \pm 0.07$ & & & 111 & & 0.7 & \pm 0.02 \\
\hline NGC 6366 & & -0. & 0 & 39 & $1.02 \pm 0.22$ & 0.005 & 0.05 & 11 & & & $23 \pm 0.01$ \\
\hline NGC 6388 & & -0.45 & 267 & 1549 & $1.30 \pm 0.03$ & $0.057 \pm 0.004$ & $0.836 \pm 0.008$ & $0.067 \pm 0.009$ & & & $88 \pm 0.03$ \\
\hline NGC 6397 & & -1.99 & 120 & 0 & $2.98 \pm 0.13$ & $0.534 \pm 0.023$ & $0.232 \pm 0.030$ & $0.008 \pm 0.011$ & 12. & $51.3 \pm 7.1$ & $29 \pm 0.17$ \\
\hline & & & 46 & 11 & & & & & & & \\
\hline NGC 6441 & & -0.44 & 243 & 1769 & $1.26 \pm 0.02$ & $0.048 \pm 0.003$ & $0.904 \pm 0.024$ & $0.081 \pm 0.022$ & 17.51 & $113.9 \pm 10.7$ & $1.55 \pm 0.03$ \\
\hline NGC 6496 & & -0.46 & 0 & 80 & $1.00 \pm 0.16$ & $0.074 \pm 0.011$ & $0.056 \pm 0.005$ & $0.021 \pm 0.006$ & 16.00 & $143.2 \pm 12.0$ & $0.11 \pm 0.02$ \\
\hline & & & 24 & 0 & & & & & & & \\
\hline NGC 6541 & & $-1.8(x-8$ & 411 & 0 & & & & & & & $10.26 \pm 0.46$ \\
\hline NGC 6584 & & & 25 & 38 & & & & & & & \\
\hline NGC 66 & & & 0 & 192 & & & & & & & \pm 0.02 \\
\hline NGC 6637 & & & 0 & 256 & \pm 0.09 & & & & & $8 \pm 11.7$ & $54 \pm 0.02$ \\
\hline NGC 6652 & & & 0 & 26 & \pm 0.28 & & & 11 & & $8 \pm 11.8$ & $64 \pm 0.01$ \\
\hline NGC 6656 & M 22 & -1 & 512 & 13 & & & & & & & \pm 0.31 \\
\hline NGC 6681 & M 70 & -1.62 & 147 & 0 & $2.97 \pm 0.11$ & $0.558 \pm 0.046$ & $334 \pm 0.045$ & $0.029 \pm 0.015$ & 15. & $5.1 \pm 6.7$ & $10.21 \pm 0.37$ \\
\hline & & -1.26 & 30 & 0 & & & & & & & \\
\hline NGC 6723 & & -1.10 & 106 & 152 & \pm 0.02 & $127 \pm 0.007$ & $04 \pm 0.010$ & $024 \pm 0.007$ & & $3.4 \pm 9.1$ & $3.38 \pm 0.08$ \\
\hline NGC 6752 & & -1.55 & 330 & 0 & $0 \pm 0.08$ & & & $042 \pm 0.004$ & & $39.4 \pm 6.3$ & $13.94 \pm 0.78$ \\
\hline & M 56 & -2.00 & 177 & 0 & & & & & & & $7.38 \pm 0.25$ \\
\hline & M 55 & & 244 & 2 & 08 & & & & & & $9 \pm 0.21$ \\
\hline NGC 6838 & M 71 & -0.8 & 0 & 70 & $1.00 \pm 0.17$ & $0.084 \pm 0.012$ & $0.057 \pm 0.010$ & $0.024 \pm 0.010$ & 14.4 & $126.5 \pm 11.2$ & $.40 \pm 0.01$ \\
\hline NGC 6934 & & -1.5 & 103 & 71 & & & & & & & $41 \pm 0.08$ \\
\hline NGC 6981 & M 72 & -1.48 & 37 & 27 & $4 \pm 0.03$ & $0.142 \pm 0.016$ & $0.570 \pm 0.019$ & $0.017 \pm 0.006$ & 16.90 & $84.9 \pm 9.2$ & $3.61 \pm 0.06$ \\
\hline & & -1.46 & 75 & 93 & & & & - & & & $.12 \pm 0.05$ \\
\hline & M 15 & -2.3 & 554 & 56 & & & & $0.069 \pm 0.006$ & & & $63 \pm 0.24$ \\
\hline NGC 7089 & M2 & -1.66 & 896 & 14 & $2.92 \pm 0.04$ & $0.150 \pm 0.035$ & $0.790 \pm 0.037$ & $0.052 \pm 0.009$ & 16.05 & $50.8 \pm 7.1$ & $8.23 \pm 0.47$ \\
\hline NGC 7099 & M 30 & -2.33 & 175 & 6 & & & & $0.022 \pm 0.010$ & & & $6.40 \pm 0.20$ \\
\hline ARP2 & & & 22 & 0 & & .021 & & - & & $64.7 \pm 8$ & $5.83 \pm 0.05$ \\
\hline IC 4499 & & -1.6 & 81 & 108 & $1.90 \pm 0.02$ & $0.113 \pm 0.026$ & $0.508 \pm 0.041$ & $0.017 \pm 0.008$ & 17.65 & $94.7 \pm 9.7$ & $2.65 \pm 0.04$ \\
\hline Lynga 7 & & -0.6 & 0 & 90 & & $0.055 \pm 0.050$ & $0.093 \pm 0.009$ & - & & 0 & \\
\hline $\mathrm{Pal} 2$ & & & 194 & 0 & $0 \pm 0.10$ & - & - & - & 20.85 & $88.2 \pm 9.4$ & $0.11 \pm 0.08$ \\
\hline Rup 106 & & -1.7 & 42 & 0 & $78 \pm 0.16$ & $135 \pm 0.012$ & $210 \pm 0.032$ & - & 17.80 & $119.2 \pm 10.9$ & $.84 \pm 0.02$ \\
\hline Terzan 7 & & & 0 & 38 & & & & - & & $133.7 \pm 11.6$ & $0.53 \pm 0.01$ \\
\hline Terzan 8 & & -2.34 & 49 & 0 & $2.92 \pm 0.19$ & $0.500 \pm 0.050$ & $0.223 \pm 0.050$ & _- & 17.95 & $55.9 \pm 7.5$ & $5.21 \pm 0.17$ \\
\hline
\end{tabular}

Notes. Columns are: observational values of blue (B), red (R) HB star counts and $H B R^{\prime}, \mathrm{L} 1, \mathrm{~L} 2$ indices, the spread in helium content $\partial Y$, V(HB) and $A_{\mathrm{CND}}(V-I)$ used to estimate the new parameter $\tau_{H B}$.

References. ${ }^{(a)}$ Harris (1996); ${ }^{(b)}$ Milone et al. (2018); ${ }^{(c)}$ Milone et al. (2014); ${ }^{(d)}$ estimated by the authors.

does not show any relevant correlation either with cluster age or with iron content.

In the figure a significant fraction of GGCs is located around the two extreme values attained by the $\mathrm{HBR}^{\prime}$ index, namely
1 (eight globulars) and 3 (ten globulars). This means that the weak sensitivity of the HBR' index does not allow us to properly trace the variation of the HB morphology when moving from metal-poor to metal-rich globulars, while the $\mathrm{HBR}^{\prime}$ index 


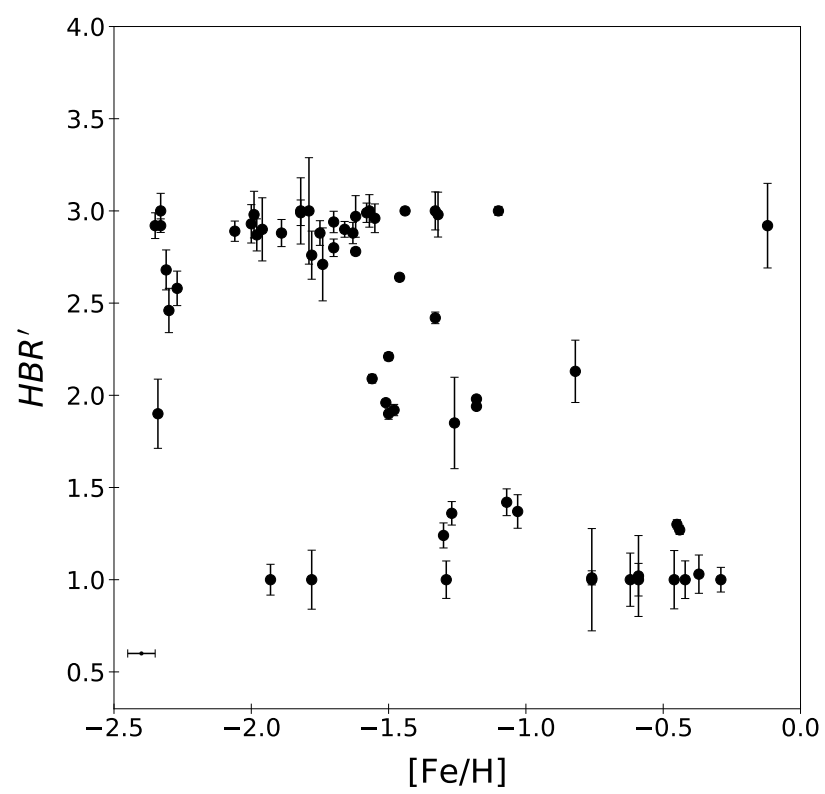

Fig. 5. Observed $\mathrm{HBR}^{\prime}$ values as a function of metal content. The metallicity scale is from Carretta et al. (2009) (see the appendix for more details). The error bar in the lower left corner gives the uncertainty of the metal content $(0.1 \mathrm{dex})$.

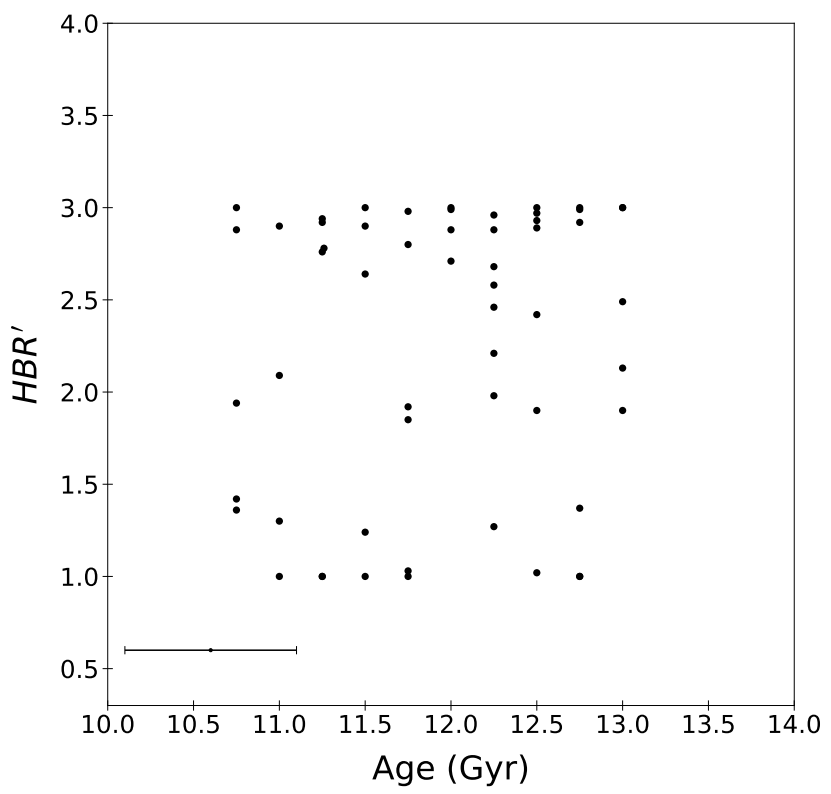

Fig. 6. Observed $\mathrm{HBR}^{\prime}$ values as a function of the cluster ages (gigayears (Gyr)) provided by VandenBerg et al. (2013), Leaman et al. (2013). The error bar in the lower left corner gives the uncertainty in the cluster ages $( \pm 0.5 \mathrm{Gyr})$.

manages to trace the variation of the HB morphology in the metalintermediate regime, where the relation appears to be almost linear. From the $\mathrm{HBR}^{\prime}$-age plane we can merely identify three different groups of clusters, with of $\mathrm{HBR}^{\prime} \sim+1, \sim+2$, and $\sim+3$, respectively.

\section{L1 and L2 HB morphology indices}

A new approach to parametrize the HB properties has been recently proposed by Milone et al. (2014). They introduced two indices, L1 and L2, and provided several possible correlations
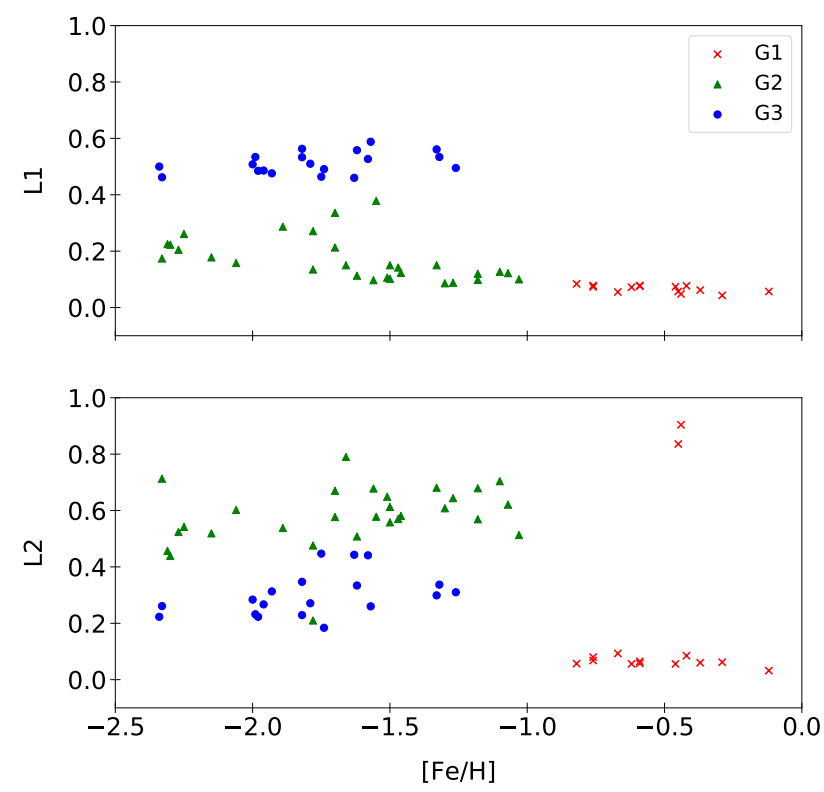

Fig. 7. L1 and L2 indices as a function of the metal content (Carretta et al. 2009). The different symbols and colours identify the different cluster groups defined in Milone et al. (2014): G1 (red crosses) for metal-rich globulars $([\mathrm{Fe} / \mathrm{H}]>-1.0), \mathrm{G} 2$ (green triangles) for clusters with $[\mathrm{Fe} / \mathrm{H}]<-1.0$ and $\mathrm{L} 1 \leq 0.4$, G3 (blue circles) for globulars with $\mathrm{L} 1 \geq 0.4$.

between the HB morphology and GGC global properties. The $\mathrm{L} 1$ index measures the distance in colour between the RGB at the same HB magnitude level and red HB stars; the L2 index is the colour extension of the HB. L1 and L2 are very easy to estimate, since their definition relies on the selection of three different points on an optical $\left(m_{F 606 W}, m_{F 606 W}-m_{F 814 W}\right)$ CMD. Since they are based on colour differences, they are independent of both cluster distance and reddening. Moreover, empirical evidence indicates that L1 and L2 correlate with intrinsic GGC properties.

Despite these advantages, the identification of the reddest HB point might be affected by the contamination of field stars since they attain similar colours. Moreover, the GGCs for which the reddest HB stars are RR Lyrae stars require additional information concerning their mean magnitudes. Furthermore, there is mounting evidence that the HB morphology changes when moving from the innermost to the outermost cluster regions (Castellani et al. 2003; Iannicola et al. 2009; Milone et al. 2014). We also note that the perceived values of both L1 and L2 might be also affected by differential reddening.

Figure 7 (similar to Fig. 2 in Milone et al. 2014) shows the new indices as a function of GGC metallicity (from Carretta et al. 2009) for the 62 GGCs in common with our sample. The GGC data set adopted by Milone et al. (2014) is similar to the current one and relies on the same ACS at HST photometric catalogues we are using for the central cluster regions. This is the reason why we decided to use the L1 and L2 values given in Table 1 of Milone et al. (2014) and listed in Cols. 7 and 8 of Table 2 together with their errors.

The data plotted in the top panel of this figure show that GGCs belonging to the G1 and the G2 groups display a steady increase of the L1 index when moving from metal-rich to metalpoor clusters. The G3 clusters attain an almost constant L1 value over more than $1 \mathrm{dex}$ in metallicity. Moreover, a glance at the data shows that the standard deviation is modest, but the correlation does not seem to be mono-parametric. Indeed, there is evidence of metal-intermediate GGCs, mainly the G2 

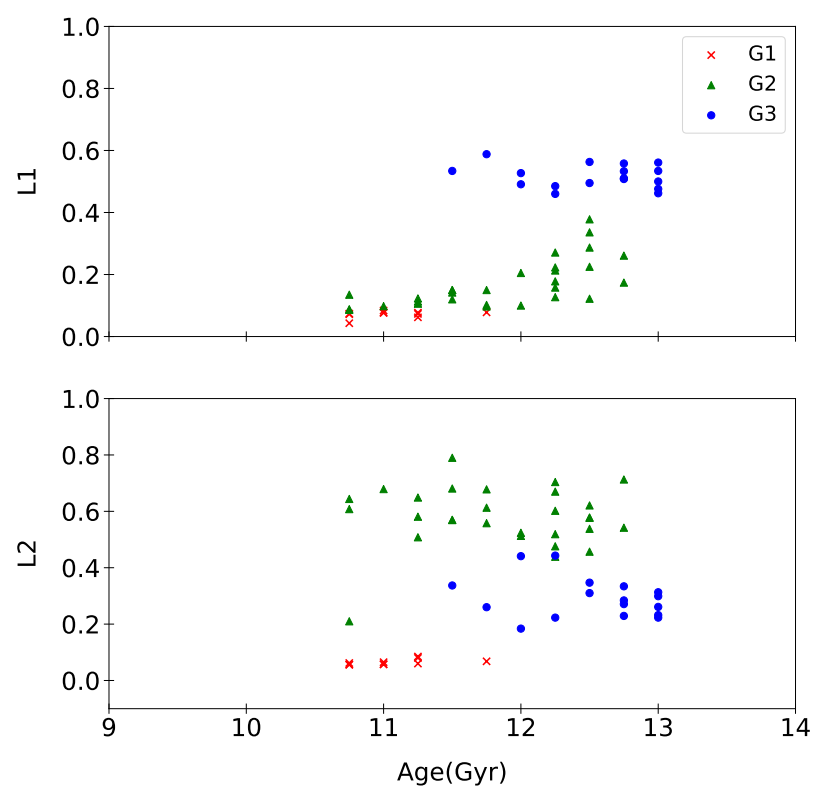

Fig. 8. L1 and L2 indices as a function of the cluster age (Gyr) from VandenBerg et al. (2013), Leaman et al. (2013). The different symbols and colours identify the different cluster groups defined in Milone et al. (2014): G1 (red crosses) for metal-rich globulars ([Fe/H] $>-1.0), \mathrm{G} 2$ (green triangles) for clusters with $[\mathrm{Fe} / \mathrm{H}]<-1.0$ and $\mathrm{L} 1 \leq 0.4$, G3 (blue circles) for globulars with $\mathrm{L} 1 \geq 0.4$.

clusters, with the same metal content but with the L1 index changing by almost a factor of two. The data plotted in the bottom panel show that the L2 index does not show a correlation with the metal content (first parameter) and indeed metal-rich clusters (G1) display a modest extent in colour, while metalintermediate and metal-poor $(\mathrm{G} 2+\mathrm{G} 3)$ clusters cover a broad colour range. There are only two exceptions: NGC 6388 and NGC 6441, the two peculiar metal-rich globulars that host red HB and blue, extreme HB stars, plus RR Lyrae stars with unusually long periods (Pritzl et al. 2003). However, the data plotted in the bottom panel do not display any relevant correlation with the metal content.

Figure 8 (similar to Fig. 5 in Milone et al. 2014) shows the correlation between the new L1 (top panel) and L2 (bottom panel) indices and the homogeneous cluster age estimates provided by VandenBerg et al. (2013), Leaman et al. (2013). The data plotted in the top panel seem to suggest a possible mild correlation between the $\mathrm{G} 2$ clusters and the absolute age. On the other hand, the G1 and the G3 clusters do not show any significant correlation with cluster age. The data plotted in this diagram clearly show that GGCs with the same age have L1 indices that change by a factor of two or three. The data plotted in the bottom panel display that the L2 index is independent of cluster age. L2 index hardly changes inside the range in age covered by G1, G2 and G3 globulars. We performed the same analysis using the GCC ages provided by Salaris \& Weiss (2002) and found similar trends. The reader interested in a more detailed analysis of the correlation between the L1/L2 indices and the GGC parameters is referred to Milone et al. (2014).

\section{A new HB morphology index: $\tau_{\mathrm{HB}}$}

To provide a new perspective on the investigation of the $\mathrm{HB}$ morphology and to overcome some of the limitations affecting the current $\mathrm{HB}$ morphology indices, we have devised a new
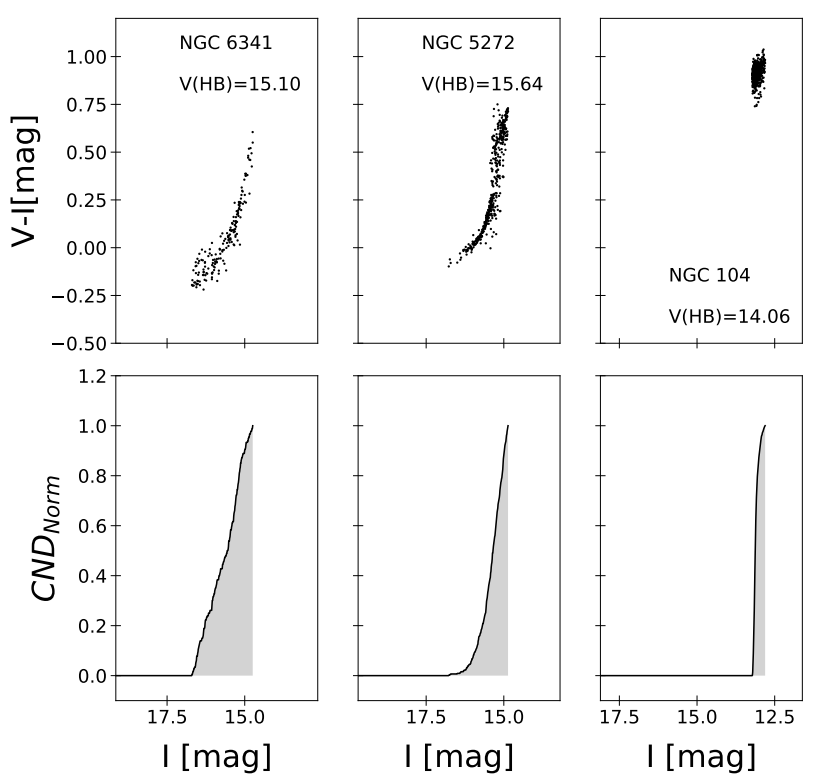

Fig. 9. Run of the normalized CND with respect to the $I$ magnitude for three globular clusters (lower panels), chosen as representative of three different metallicity regimes (NGC 6341, NGC 5272, and NGC 104). Upper panels: horizontal branch of the three chosen globulars in $I, V-I$ CMDs.

parameter, christened $\tau_{\mathrm{HB}}$, based on the ratio of the areas subtended by the CNDs as a function of magnitude ( $I$-band) and colour $(V-I)$ of HB stars. To estimate the new HB morphology index first we defined a box in the $I, V-I$ CMD large enough to include all cluster HB stars, independently of the HB morphology. Then for each cluster we computed the CND in both magnitude and in colour. The reference luminosity level we selected from the Harris catalogue is the $V(\mathrm{HB})$, which is the mean visual magnitude of HB stars inside the RR Lyrae instability strip. This $V$ magnitude was transformed into an $I$-band magnitude assuming a mean $V-I$ colour for the RR Lyrae stars equal to 0.45 (Braga et al. 2016). To perform homogeneous star counts ranging from EHB to RHB stars, we defined a box covering $1.7 \mathrm{mag}$ in $V-I$ colour and $6.5 \mathrm{mag}$ in $I$-band, which is 4.5 mag fainter and 2.0 mag brighter than the $I$-band HB luminosity level. Finally, the selected HB stars are sorted as a function of the $I$-band apparent magnitude and we cumulated the star counts starting from the faintest and reddest stars in the box.

The top panels of Fig. 9 display HB stars in the $I, V-I$ CMD for three GGCs covering a broad range of iron abundances and HB morphologies. They are NGC $6341([\mathrm{Fe} / \mathrm{H}]=-2.25)$, with an HB dominated by hot and extreme blue HB stars, NGC $5272([\mathrm{Fe} / \mathrm{H}]=-1.50)$ with a $\mathrm{HB}$ that includes blue and red HB stars, as well as RR Lyrae stars, and finally NGC 104 $([\mathrm{Fe} / \mathrm{H}]=-0.76)$ which is characterized by a red HB morphology. The bottom panels of the same figure display the normalized I-band CNDs of the HB stars plotted in the top panel. The data plotted in these panels show that the area subtended by the three CNDs ( $A_{\mathrm{CND}}$, shaded area) changes significantly when moving from an HB morphology dominated by hot and extreme HB stars, to a morphology dominated by red HB stars. We find a slightly constant decrease of the $I$-band $A_{\mathrm{CND}}$ by a factor of $\sim 1.4$ when moving from NGC $6341\left(A_{\mathrm{CND}}(I)=424.2\right)$ to NGC 5272 $\left(A_{\mathrm{CND}}(I)=336.6\right)$ to NGC $104\left(A_{\mathrm{CND}}(I)=217.9\right)$. A decrease on the order of approximately two when moving from an extreme blue HB morphology (NGC 6341) to a red one (NGC 104). 

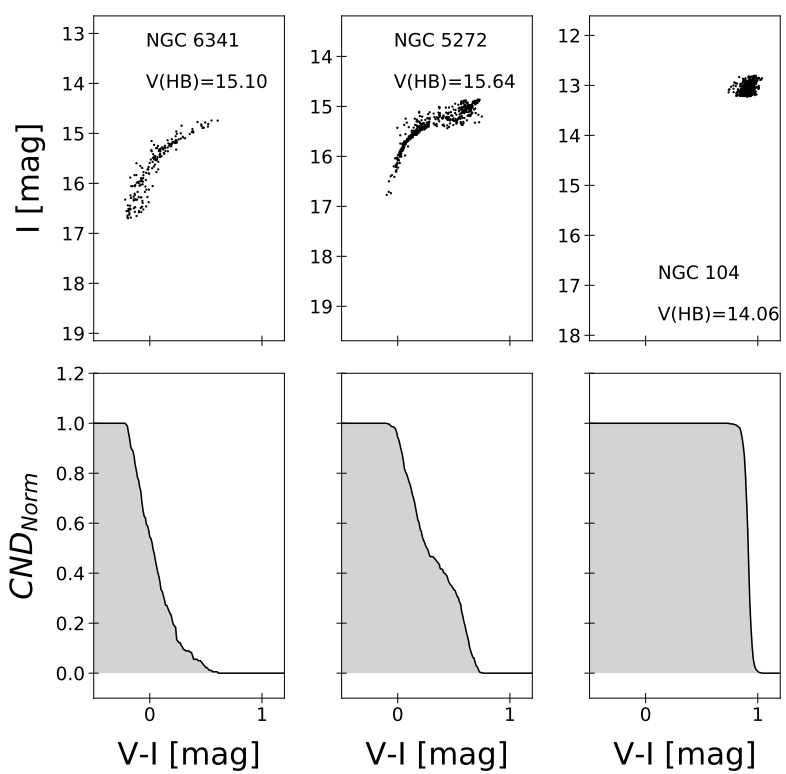

Fig. 10. Run of the normalized CND with respect to $V-I$ for the same three globular clusters (lower panels) of Fig. 9. Upper panels: horizontal branch of the three chosen globulars in $I, V-I$ CMDs.

Then, we sorted the HB stars located inside the box as a function of the $(V-I)$ colour. The apparent colours were de-reddened using the colour excesses $(E(B-V))$ listed in Table 1 . In this case the HB stars were counted starting from the reddest (coldest) HB stars. The top panels of Fig. 10 display HB stars in the $I, V-I$ CMD for the same GGCs as in Fig. 9. The bottom panels display the normalized $V-I$ CND of the HB stars plotted in the top panel. The shaded area shows a stronger sensitivity compared with the $I$-band $A_{\mathrm{CND}}$; indeed, they increase by almost a factor of three when moving from NGC $6341\left(A_{\mathrm{CND}}(V-I)=47.4\right)$ to $\operatorname{NGC} 5272\left(A_{\mathrm{CND}}(V-I)=81.5\right)$ and to NGC $104\left(A_{\mathrm{CND}}(V-I)=\right.$ 137.9).

To estimate the CNDs in magnitude and in colour we accounted for the entire sample of HB stars, meaning stars located inside the RRL instability strip were included. The spread in magnitude and in colour of these objects is larger compared with typical HB stars. The difference is intrinsic, since we are using the mean of the measurements in both the $V$ and the $I$ band. In other words, we did not perform an analytical fit of the phased measurements.

To investigate the difference between the classical (HBR') and the new $\left(\tau_{\mathrm{HB}}\right)$ morphology index on a more quantitative basis, the left panel of Fig. 11 shows the comparison between the $I$-band $A_{\mathrm{CND}}$ and the $\mathrm{HBR}^{\prime}$ index. The data plotted in this panel display a very well-defined linear correlation between $I$-band $A_{\mathrm{CND}}$ and $\mathrm{HBR}^{\prime}$ index in the regime in which the latter attains intermediate values. The saturation for clusters dominated by blue $\mathrm{HB}$ morphologies $\left(\mathrm{HBR}^{\prime} \sim 3\right)$ and red $\mathrm{HB}$ morphologies $\left(\mathrm{HBR}^{\prime} \sim 1\right)$ is also quite clear at the top and the bottom of the panel. The right panel shows the same comparison as the left panel, but for the $V-I A_{\mathrm{CND}}$. The data plotted in this panel show a linear anti-correlation over the entire range of values attained by the HBR' index.

We estimated the ratio between the area covered by the CND in magnitude and in colour, namely

$\tau_{\mathrm{HB}}=\frac{A_{\mathrm{CND}}(I)}{A_{\mathrm{CND}}(V-I)}$.
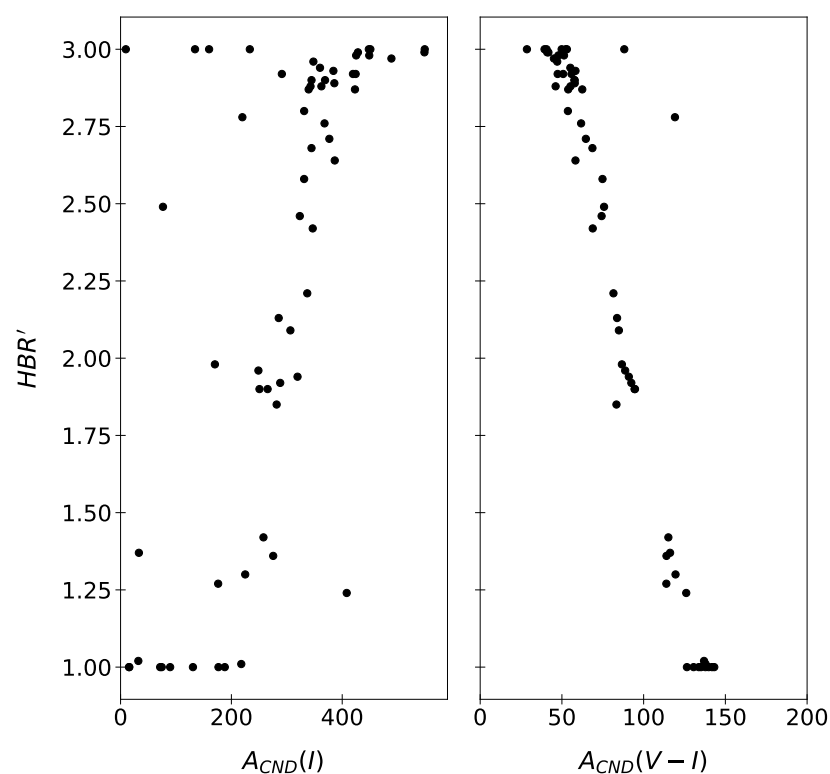

Fig. 11. Indices $A_{\mathrm{CND}}(I)$ and $A_{\mathrm{CND}}(V-I)$ vs. the $\mathrm{HBR}^{\prime}$ morphology index.

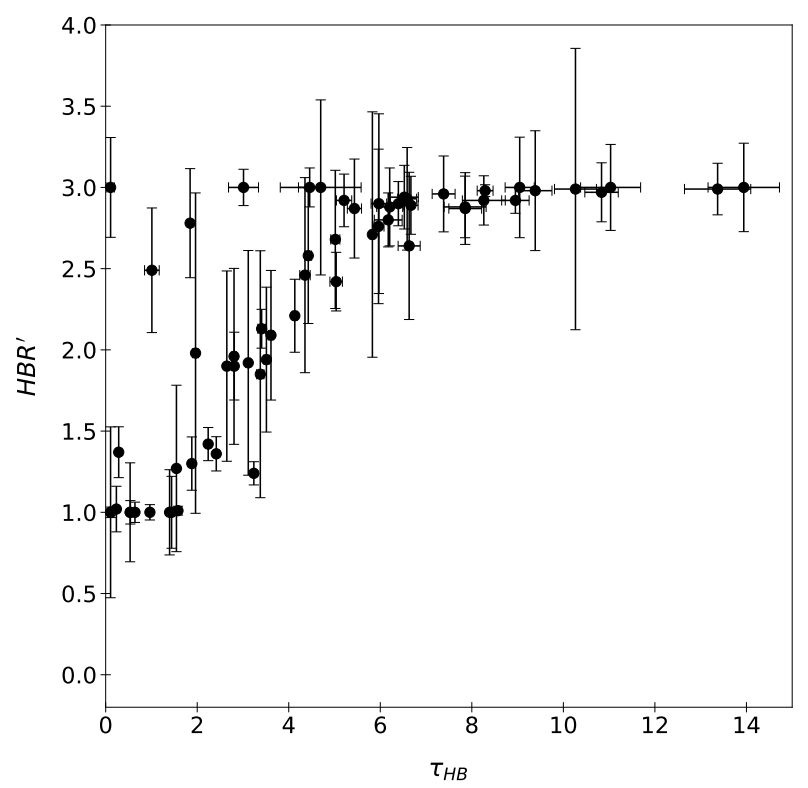

Fig. 12. Our new HB morphology index $\tau_{\mathrm{HB}}$ vs. HBR'.

The $V$ magnitude levels of the $\mathrm{HB}$, which we transformed to $I(\mathrm{HB})$ using the mean $V-I$ of $0.45 \mathrm{mag}, A_{\mathrm{CND}}(V-I), \tau_{\mathrm{HB}}$, and their errors for the GGCs in our sample are listed in Cols. 10, 11, and 12 of Table 2, respectively. We propagated the error on $\tau_{\mathrm{HB}}$ considering Poissonian uncertainties in the $A_{\mathrm{CND}}$.

Although $\tau_{\mathrm{HB}}$ estimate is slightly more complex than the other indices presented in the literature, the new HB morphology index presents several key advantages compared with the classical HBR' and L1/L2 indices:

Dynamical range. The current sample covers a range in $\tau_{\mathrm{HB}}$ that is a factor of seven larger than the range covered by the $\mathrm{HBR}^{\prime}$ index. The data plotted in Fig. 12 show that when moving from metal-rich to less metal-rich GGCs $(-1.0<[\mathrm{Fe} / \mathrm{H}]<0)$ the new index changes from zero to roughly three while the old one changes from one to roughly 1.5 . The correlation between the old and new indices is linear in the metal intermediate regime, 

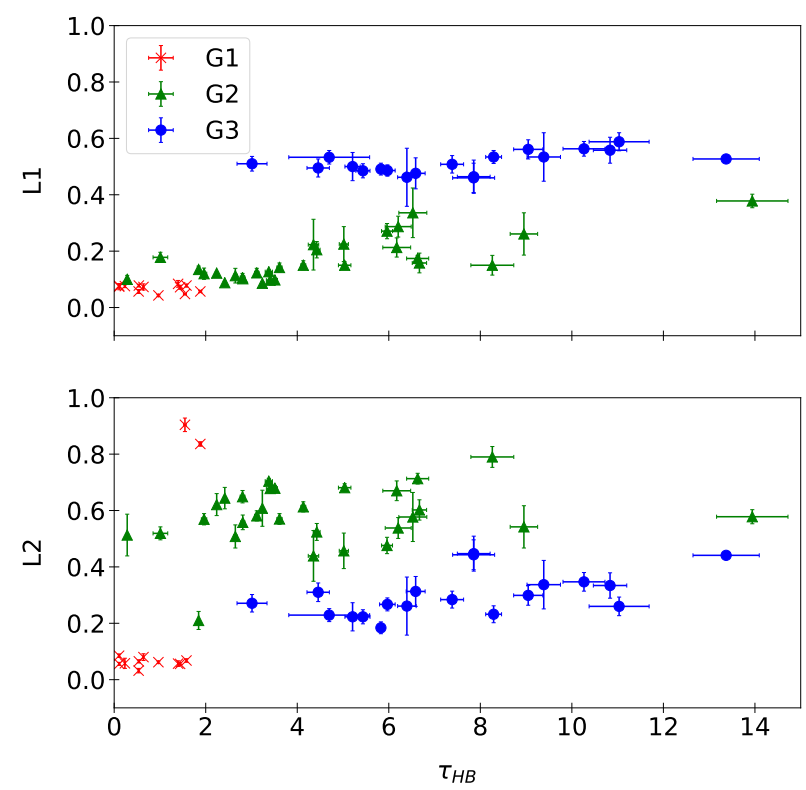

Fig. 13. L1 (top) and L2 (bottom) indices as a function of $\tau_{\mathrm{HB}}$ for the globulars in our sample. The different symbols and colours identify the different cluster groups defined in Milone et al. (2014): G1 (red crosses) for metal-rich globulars $([\mathrm{Fe} / \mathrm{H}]>-1.0), \mathrm{G} 2$ (green triangles) for clusters with $[\mathrm{Fe} / \mathrm{H}]<-1.0$ and $\mathrm{L} 1 \leq 0.4, \mathrm{G} 3$ (blue circles) for globulars with $\mathrm{L} 1 \geq 0.4$.

but it degenerates in the more metal-poor regime. Indeed, the $\tau_{\mathrm{HB}}$ index increases by more than a factor of two (from six to fourteen), whereas the $\mathrm{HBR}^{\prime}$ takes on an almost constant value of three.

The variation in $\tau_{\mathrm{HB}}$ is a factor of twenty larger than the range covered by the L1 and L2 indices. The data plotted in Fig. 13 show that the G3 group is characterized by L1 values that are almost constant $(\mathrm{L} 1 \sim 0.5)$, while $\tau_{\mathrm{HB}}$ changes from three to thirteen. The G1 and G2 groups display a mild linear correlation between $\mathrm{L} 1$ and $\tau_{\mathrm{HB}}$, but once again the variation in the $\mathrm{L} 1$ index is modest when compared with $\tau_{\mathrm{HB}}$ index $(0.3 \mathrm{vs}$. 7$)$. The correlation between $\mathrm{L} 2$ and $\tau_{\mathrm{HB}}$ is more noisy with a large spread at fixed $\tau_{\mathrm{HB}}$ value.

Detailed sampling of the HB morphology. The CND in both magnitude and colour is sensitive to the star distribution along the HB. Data listed in Table 2 show that two metal-poor clusters, NGC 4833 and NGC 6341, with similar HBR' $(2.88 \pm 0.07$ vs. $2.92 \pm 0.07)$ and $\mathrm{L} 1(0.287 \pm 0.037$ vs. $0.261 \pm 0.075)$ indices attain $\tau_{\mathrm{HB}}$ values that differ at the $50 \%$ level $(6.21 \pm 0.21$ vs. $8.95 \pm 0.30$ ). The same applies to metal-rich clusters, and indeed, two clusters like NGC 104 and NGC 6637 have the same $\mathrm{HBR}^{\prime}(1.01 \pm 0.04$ vs. $1.00 \pm 0.09)$ and $\mathrm{L} 1(0.078 \pm 0.005$ vs. $0.078 \pm 0.004)$ values, but the $\tau_{\mathrm{HB}}$ value of the former cluster is a factor of three larger than the latter one $(1.58 \pm 0.01 \mathrm{vs}$. $0.54 \pm 0.02)$. Data listed in Table 3 highlight several other pairs of GGCs characterized by a very similar iron content (Col. 2), $\mathrm{HBR}^{\prime}$ (Col. 3), and $\mathrm{L1}^{4}$ (Col. 4) value, but quite different $\tau_{\mathrm{HB}}$ values (Col. 5). The various pairs are separated in the table with horizontal lines.

Global star count of HB stars. The $\tau_{\mathrm{HB}}$ does not require any identification of specific subgroups (blue, red, variables).

\footnotetext{
4 We did not consider L2 since it does not correlate with age, metallicity, and $\tau_{\mathrm{HB}}$.
}

Table 3. Examples of globular pairs of similar metallicity and $\mathrm{HBR}^{\prime}$, but different values in $\tau_{\mathrm{HB}}$.

\begin{tabular}{ccccc}
\hline \hline ID & {$[\mathrm{Fe} / \mathrm{H}]$} & $\mathrm{HBR}^{\prime}$ & $\mathrm{L} 1$ & $\tau_{\mathrm{HB}}$ \\
\hline NGC 0104 & $-0.76 \pm 0.02$ & $1.01 \pm 0.04$ & $0.078 \pm 0.005$ & $1.58 \pm 0.01$ \\
NGC 6652 & $-0.76 \pm 0.14$ & $1.00 \pm 0.28$ & $0.073 \pm 0.011$ & $0.64 \pm 0.01$ \\
NGC 6121 & $-1.18 \pm 0.02$ & $1.98 \pm 0.01$ & $0.120 \pm 0.020$ & $1.96 \pm 0.06$ \\
NGC 1851 & $-1.18 \pm 0.08$ & $1.95 \pm 0.01$ & $0.098 \pm 0.004$ & $3.51 \pm 0.06$ \\
NGC 6752 & $-1.55 \pm 0.01$ & $3.00 \pm 0.08$ & $0.378 \pm 0.024$ & $13.94 \pm 0.78$ \\
NGC 6934 & $-1.56 \pm 0.09$ & $2.13 \pm 0.02$ & $0.097 \pm 0.0013$ & $3.41 \pm 0.08$ \\
NGC 6144 & $-1.82 \pm 0.05$ & $3.00 \pm 0.18$ & $0.533 \pm 0.024$ & $4.98 \pm 0.88$ \\
NGC 6541 & $-1.82 \pm 0.08$ & $2.99 \pm 0.07$ & $0.563 \pm 0.026$ & $10.26 \pm 0.46$ \\
\hline
\end{tabular}

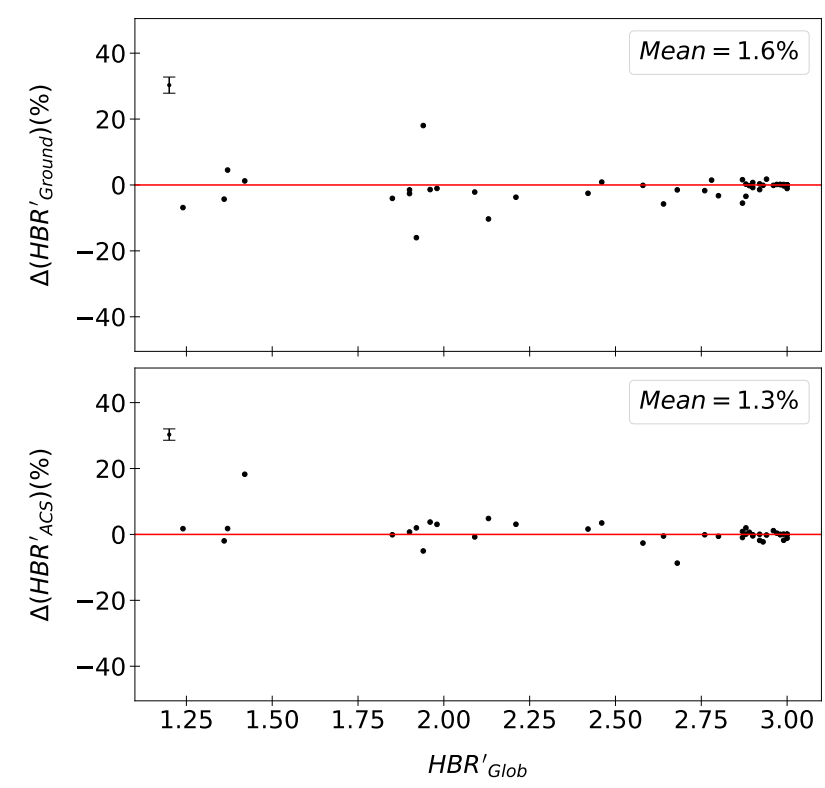

Fig. 14. Top: relative difference between the global $\mathrm{HBR}^{\prime}$ and the $\mathrm{HBR}^{\prime}$ values only based on ground-based data versus the global index. Note that in the estimate of the global index the priority in selecting the photometry was given to space-based (ACS at HST) data. Bottom: as the top panel, but the relative difference is between the global index and the $\mathrm{HBR}^{\prime}$ index only based on space-based data. The standard deviation of the estimates is represented by the error bar at the top left corner of the panels. At the top right corners we display the mean relative difference.

\section{Comparison between space- and ground-based data}

As described in Sect. 2, when available we preferred the spacebased observations for our analysis, while we used the groundbased data for the outer regions of the globulars in our sample. In this section, we analyse the $\mathrm{HBR}^{\prime}$ and $\tau_{\mathrm{HB}}$ index evaluations using either ACS or ground-based observations, comparing them to the "global" index, which is the index estimated from both space- and ground-based data (see Sects. 3 and 5), giving higher priority to the first ones when both measures where available.

Figure 14 shows the relative difference between the HBR' index estimated by using either ground-based (top panel) or ACS (bottom panel) data as a function of the global value listed in Col. 6 of Table 2. Data plotted in this figure show the following results. The top panel of Fig. 14 shows that the relative difference in $\mathrm{HBR}^{\prime}$ index from ground-based data is on average approximately $1.6 \%$. The major exceptions are represented by the globulars NGC $1851\left(\left|\Delta\left(\mathrm{HBR}^{\prime}{ }_{\text {Ground }}\right)\right| \sim 18 \%\right)$ and NGC $7006\left(\mid \Delta\left(\right.\right.$ HBR $\left.\left._{\text {Ground }}\right) \mid \sim 16 \%\right)$. The difference is mainly 


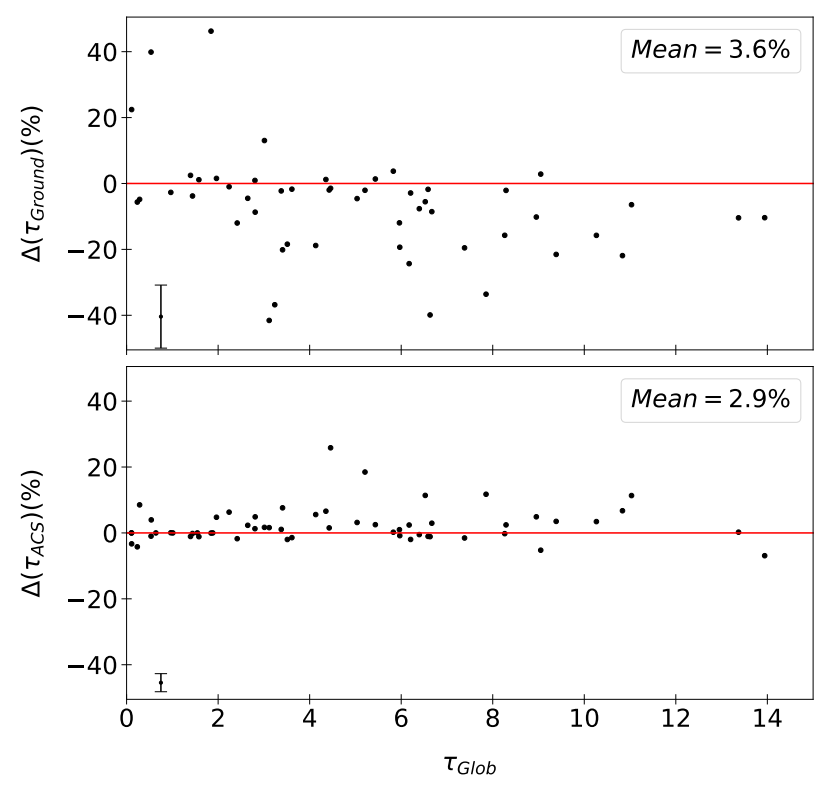

Fig. 15. Top: relative difference between the global $\tau_{\mathrm{HB}}$ and the $\tau_{\mathrm{HB}}$ index only based on ground-based data versus the global index. Note that in the estimate of the global index the priority in selecting stars was given to space-based (ACS at HST) data. Bottom: as the top panel, but the relative difference is between the global index and the $\tau_{\mathrm{HB}}$ index only based on space-based data. The standard deviation of the estimates is represented by the error bar at the bottom left corner of the panels. At the top right corners we display the mean relative difference.

caused by the star counts of red HB stars (R) from ground-based observations. They provide a lower contribution when compared with space-based data $\left(\mathrm{HBR}^{\prime}{ }_{\text {Ground }} \sim 2.28\right.$ vs. HBR $_{\text {Global }} \sim 1.94$ for the former cluster, $\mathrm{HBR}^{\prime}{ }_{\text {Ground }} \sim 1.61 \mathrm{vs} . \mathrm{HBR}^{\prime}{ }_{\text {Global }} \sim 1.92$ for the latter one).

The bottom panel shows that the difference in $\mathrm{HBR}^{\prime}$ index between $\mathrm{HBR}^{\prime}{ }_{\mathrm{ACS}}$ and $\mathrm{HBR}_{\text {Global }}$ is on average $\sim 1.3 \%$. It is marginally lower than the difference based on ground-based data. The single exception is NGC 6362, which is characterized by $\left|\Delta\left(\mathrm{HBR}^{\prime}{ }_{\mathrm{ACS}}\right)\right| \sim 18 \%$. Its global $\mathrm{HBR}^{\prime}$ index turns out to be redder than the ACS one $\left(\mathrm{HBR}^{\prime}{ }_{\mathrm{ACS}} \sim 1.42 \mathrm{vs} . \mathrm{HBR}^{\prime}{ }_{\mathrm{Global}} \sim 1.68\right)$ since in the global $\mathrm{HBR}^{\prime}$ estimate the contribution of red $\mathrm{HB}$ stars is mainly given by red HB stars from ground-based observations.

Figure 15 shows the global $\tau_{\mathrm{HB}}$ index, evaluated in Sect. 5, versus the relative difference between the $\tau_{\mathrm{HB}}$ index based either on ground-based (top panel) or on ACS (bottom panel) observations and the global one. A glance at the data plotted in this figure discloses a couple of interesting findings. The top panel shows that the relative difference in $\tau_{\mathrm{HB}}$ index based on ground-based data shows a larger dispersion compared to that from space-based (bottom panel) data. The mean relative difference between $\tau_{\mathrm{HB}, \text { Global }}$ and $\tau_{\mathrm{HB}, \text { Ground }}$ is $\sim 3.6 \%$. Moreover, thirteen globulars have a difference of $\left|\Delta\left(\tau_{\mathrm{HB}, \text { Ground }}\right)\right|$ greater than $20 \%$. In this context it is worth mentioning that amongst them eleven are small, concentrated globulars. Indeed, their half-mass radius $r_{\mathrm{h}}$ (Harris 1996) is entirely located inside the ACS FoV, namely NGC 1851 (23\%, $\left.r_{\mathrm{h}}=0.51^{\prime}\right), \operatorname{NGC} 2298\left(24 \%, r_{\mathrm{h}}=0.98^{\prime}\right), \operatorname{NGC} 5286\left(32 \%, r_{\mathrm{h}}=\right.$ $\left.0.73^{\prime}\right), \operatorname{NGC} 5986\left(51 \%, r_{\mathrm{h}}=0.98^{\prime}\right), \operatorname{NGC} 6681\left(28 \%, r_{\mathrm{h}}=\right.$ $\left.0.71^{\prime}\right), \operatorname{NGC} 6779\left(24 \%, r_{\mathrm{h}}=1.1^{\prime}\right), \operatorname{NGC} 6934\left(25 \%, r_{\mathrm{h}}=0.69^{\prime}\right)$, NGC $7006\left(71 \%, \quad r_{\mathrm{h}}=0.44^{\prime}\right), \quad$ NGC $7078\left(66 \%, \quad r_{\mathrm{h}}=1.0^{\prime}\right)$, Rup $106\left(32 \%, r_{\mathrm{h}}=1.05^{\prime}\right)$, and Terzan $7\left(29 \%, r_{\mathrm{h}}=0.77^{\prime}\right)$. This means that the main contribution in the $\tau$ index comes from space-based data, while ground-based data mainly contribute for

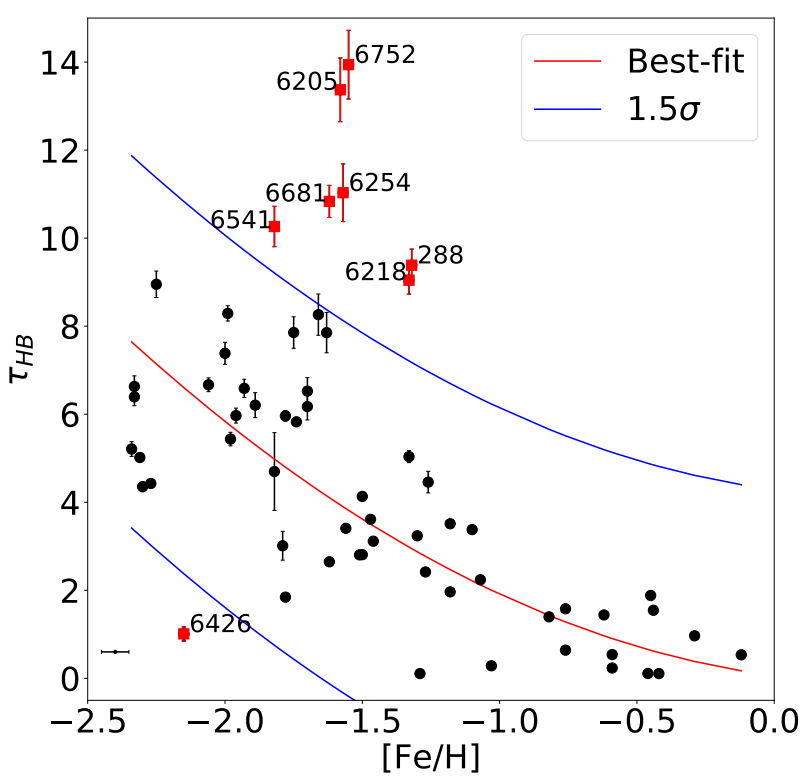

Fig. 16. Variation of $\tau_{\mathrm{HB}}$ as a function of cluster metallicity (Carretta et al. 2009). The red line shows the quadratic best fit, the blue lines show $1.5 \sigma$ levels. Red squares display the outliers, which are those objects located at more than $1.5 \sigma$ from the quadratic fit. In the left corner the error bar shows the 0.1 dex error on the metal content.

HB stars located in the cluster outskirts. Two out of the thirteen globulars are larger, but located at large distances (true distance modulus DM $\sim 15 \mathrm{mag})$, namely NGC $288\left(27 \%, r_{\mathrm{h}}=2.23^{\prime}\right)$ and NGC $5272\left(27 \%, r_{\mathrm{h}}=2.31^{\prime}\right)$. This means that, even in these cases, most of the cluster stars are located inside the ACS FoV, giving a major contribution to the $\tau_{\mathrm{HB}, \text { Global }}$ when compared to the ground-based observations.

The bottom panel shows that the relative variation between $\tau_{\mathrm{HB}, \text { Global }}$ and $\tau_{\mathrm{HB}, \mathrm{ACS}}$ is, as expected low, on average $2.9 \%$. The only exception is NGC $6717(26 \%)$, in which ACS data do not populate the brighter region of the $\mathrm{HB}$, giving a different estimate of $\tau_{\mathrm{HB}, \mathrm{ACS}}$ compared to the global one (5.61 vs. 4.46).

These findings support the fact that both ground-based and space observations of our sample of GGCs are statistically consistent and reliable. Moreover, our estimates of $\mathrm{HBR}^{\prime}$ and $\tau_{\mathrm{HB}}$ are valid and well grounded: independently of the choice about the origin of the data they give the same results within a difference of at most $20 \%$ for $\tau_{\mathrm{HB}}$ estimates.

The evidence of a larger relative difference in the $\tau_{\mathrm{HB}}$ index shows that potentially our new index could quantify the different possible contribution to the HB morphology given by inner and outer populations within the globulars. This difference between star counts based on ground-based and on space-based data will be addressed in a future paper focused on the spectral energy distribution of Galactic globulars.

\section{Correlation of $\tau_{\mathrm{HB}}$ with cluster metallicity, age, and helium content}

To investigate the correlation between $\tau_{\mathrm{HB}}$ and fundamental cluster parameters, we study its relationship to cluster metallicity, absolute age, and internal spread in Helium abundance. Figure 16 shows $\tau_{\mathrm{HB}}$ as a function of the iron content. The bulk of metal-poor GGCs $([\mathrm{Fe} / \mathrm{H}] \leq-1.5)$ attains $\tau_{\mathrm{HB}}$ values ranging from $\sim 9$ to $\sim 4$. In the metal-intermediate regime 

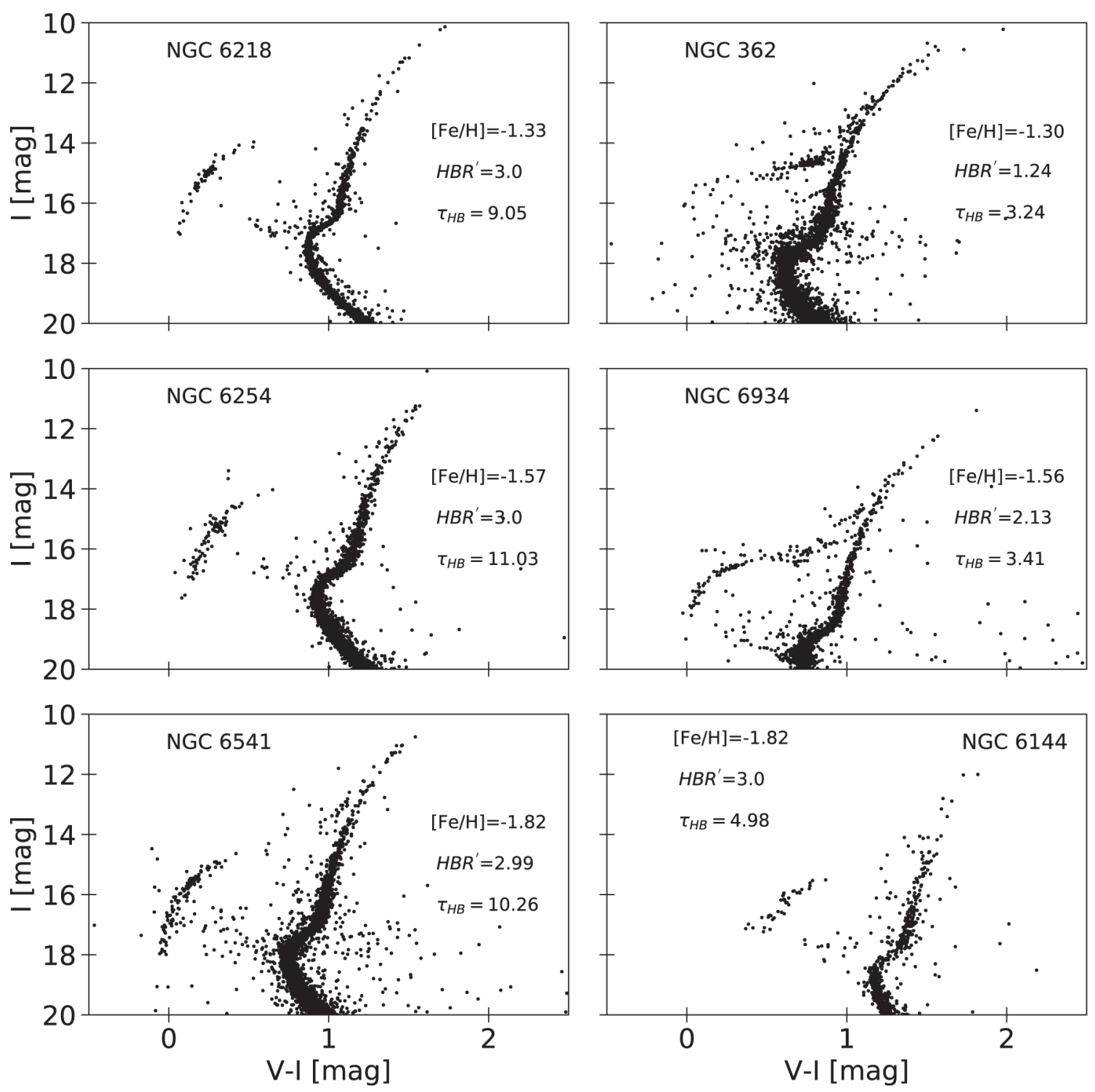

Fig. 17. CMDs $(I, V-I)$ for three pairs of GGCs in the sample. Left panels: clusters belonging to the outlier group. Right panels: CMDs of clusters with similar $[\mathrm{Fe} / \mathrm{H}]$ as the outlier ones, but following the main $\tau_{\mathrm{HB}}-[\mathrm{Fe} / \mathrm{H}]$ relation.

$(-1.5 \leq[\mathrm{Fe} / \mathrm{H}] \leq-1.0)$ the bulk of the GGCs are characterized by $\tau_{\mathrm{HB}}$ ranging from $\sim 5$ to $\sim 2$, while metal-rich clusters $([\mathrm{Fe} / \mathrm{H}] \geq-1.0)$ attain $\tau_{\mathrm{HB}}$ values smaller than 2 on average. There is a small sample of GGCs that attains $\tau_{\mathrm{HB}}$ values that are, at fixed metal content, either systematically larger or systematically smaller than the typical ranges. To investigate on a more quantitative basis the identification of these GGCs, we performed a quadratic fit of the bulk of the GGCs. We found the best-fit function

$\tau_{\mathrm{HB}}=0.06-0.83 \cdot[\mathrm{Fe} / \mathrm{H}]+1.03 \cdot[\mathrm{Fe} / \mathrm{H}]^{2}$,

with a $\sigma=3.08$ dispersion (see the red line in Fig. 16). We have defined as "outliers" the GGCs that attain, at fixed metal content, $\tau_{\mathrm{HB}}$ values that are more than $1.5 \sigma$ away from the best quadratic fit. We ended up with a subsample of seven metalpoor and metal-intermediate $(-1.82 \leq[\mathrm{Fe} / \mathrm{H}] \leq-1.32)$ GGCs with $\tau_{\mathrm{HB}}$ values larger than 9 (see red squares in Fig. 16). This subsample includes the GGCs that attain the largest $\tau_{\mathrm{HB}}$ values, namely NGC $6205\left(\tau_{\mathrm{HB}}=13.37 \pm 0.72\right)$ and NGC 6752 $\left(\tau_{\mathrm{HB}}=13.94 \pm 0.78\right)$. This means that they are characterized by very blue HB morphologies. Data plotted in Fig. 16 also show a metal-poor GGC (NGC 6426) with $\tau_{\mathrm{HB}}=1.01 \pm 0.16$ that is a factor of four to ten smaller than the bulk of GGCs with similar metal abundances.

The above empirical evidence indicates that the new $\mathrm{HB}$ morphology index appears to be a solid diagnostic to select GGCs that are strongly affected by the second parameter problem. To further investigate the nature of these clusters we decided to perform a more detailed analysis of the GGCs in the metallicity range covered by the second parameter clusters. We selected three out of the eight outliers, namely NGC $6218([\mathrm{Fe} / \mathrm{H}]=-1.33$, Age $=$ $13 \mathrm{Gyr})$, NGC $6254([\mathrm{Fe} / \mathrm{H}]=-1.57, \quad$ Age $=11.75 \mathrm{Gyr})$, and NGC $6541([\mathrm{Fe} / \mathrm{H}]=-1.82$, Age $=12.50 \mathrm{Gyr})$, covering roughly 0.5 dex in metal content. The left panels of Fig. 17 show the $I$, $V-I$ CMDs of these clusters. Assuming that the HB morphology is mainly driven by a difference in metal content, we selected three GGCs with iron abundances very similar to the selected outliers, but with $\tau_{\mathrm{HB}}$ values close to the best-fit line plotted in Fig. 16, namely NGC $362([\mathrm{Fe} / \mathrm{H}]=-1.30$, Age $=10.75 \mathrm{Gyr})$, NGC $6934([\mathrm{Fe} / \mathrm{H}]=-1.56$, Age $=11.75 \mathrm{Gyr})$, and NGC 6144 $([\mathrm{Fe} / \mathrm{H}]=-1.82$, Age $=12.75 \mathrm{Gyr})$. The right panels of Fig. 17 


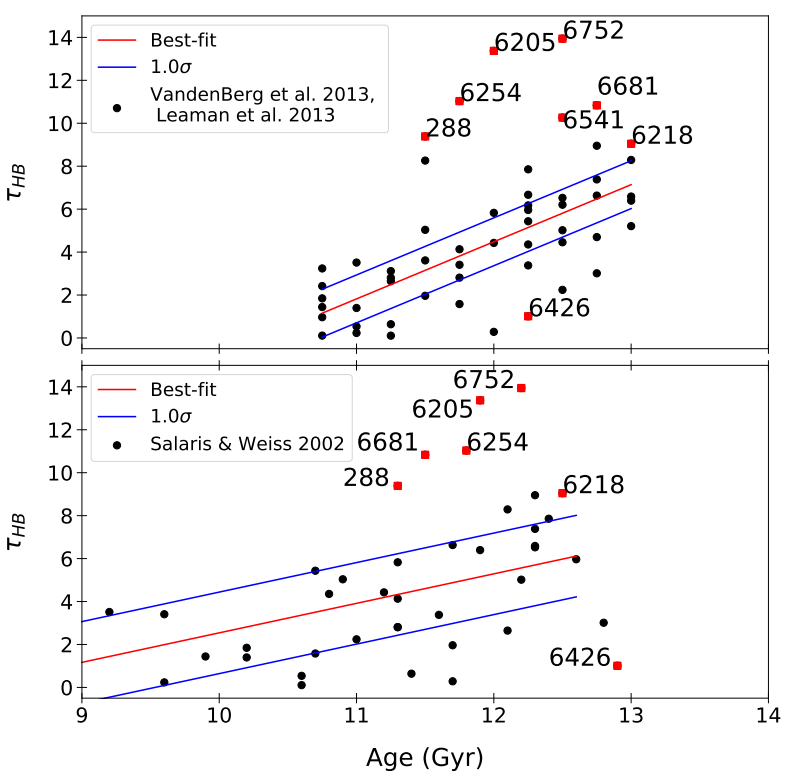

Fig. 18. Our index $\tau_{\mathrm{HB}}$ as a function of cluster ages obtained from different authors. Top: cluster ages from VandenBerg et al. (2013), Leaman et al. (2013). The error bar in the lower left corner shows the conservative \pm 0.5 Gyr error on the GGC ages. Bottom: ages from Salaris \& Weiss (2002) based on the metallicity scale provided by Carretta \& Gratton (1997). In both panels red squares are the same second parameter clusters shown in Fig. 16 and discussed in this section. Red lines determine the best-fit to each data sample, blue lines the $\pm 1 \sigma$ levels.

display their $I, V-I C M D$ s. The CMDs display several interesting features worth discussing.

The top panels of Fig. 17 compare the CMDs of two metalintermediate GGCs: an outlier cluster characterized by a very blue HB morphology, and a typical one (in terms of $\tau_{\mathrm{HB}}, 9.05 \mathrm{vs}$. 3.24) , mainly dominated by red HB stars. It is worth mentioning that the HB morphology of this pair of clusters could also be traced on the basis of the HBR' index. Indeed, the HBR' value of NGC 6218 is a factor of 2.5 larger than the $\mathrm{HBR}^{\prime}$ value of NGC 362.

The middle panels of Fig. 17 also display the CMDs of two metal-intermediate GGCs. The outlier GGC is characterized by a very blue HB morphology, while the typical one shows an HB that hosts variable stars, blue and red HB stars. For these GGCs the $\mathrm{HBR}^{\prime}$ index decreases by only the $30 \%$ when moving from NGC 6254 (3.00) to NGC 6934 (2.13). Interestingly enough, the $\tau_{\mathrm{HB}}$ index differs by more than a factor of three.

The bottom panels of Fig. 17 display the CMDs of two metalpoor GGCs. The outlier GGC shows, as expected, a very blue HB morphology, while the "typical" one also shows a blue HB morphology. The HBR' index for these two GGCs is identical within the errors: 2.99 for NGC 6541 and 3.00 for NGC 6144. On the other hand, the $\tau_{\mathrm{HB}}$ index differs by more than a factor of two (10.26 vs. 4.98).

The current findings further support the strong sensitivity of the $\tau_{\mathrm{HB}}$ index to variations in $\mathrm{HB}$ morphology when moving from the metal-intermediate to the metal-poor regime.

The anti-correlation found between the $\tau_{\mathrm{HB}}$ index and the cluster metallicity does not include information about the cluster age. We have then investigated whether the new HB morphology index is correlated with the cluster ages.

To establish the age dependence we took advantage of the recent homogeneous age estimates provided by VandenBerg et al. (2013), Leaman et al. (2013). The data plotted in the top
Table 4. Best fit parameters for the linear functions fitting the $\tau_{\mathrm{HB}}$-Age relations used in this work.

\begin{tabular}{lccc}
\hline \hline & $a$ & $b$ & $\sigma$ \\
\hline VandenBerg + Leaman et al. & $-27.23 \pm 6.34$ & $2.69 \pm 0.53$ & 1.21 \\
Salaris and Weiss & $-11.20 \pm 3.55$ & $1.37 \pm 0.32$ & 1.89 \\
\hline
\end{tabular}

panel of Fig. 18 display a well-defined linear correlation between age and $\tau_{\mathrm{HB}}$. In particular, there is evidence that, when moving from a red to a blue HB morphology, GGCs become on average older. We performed a linear fit ( $\tau_{\mathrm{HB}}=a+b \cdot$ Age, red line) whose coefficients are listed in Table 4, together with the standard deviation $(\sigma)$. The dispersion around the linear fit is modest, equal to 1.21. However, the second parameter globulars identified in the $\tau_{\mathrm{HB}}-[\mathrm{Fe} / \mathrm{H}]$ plane do not follow the same trend. They are on average more than $1.5 \sigma$ away from the main relation, that is, at fixed cluster age their $\tau_{\mathrm{HB}}$ values are systematically larger than typical GGCs. This further supports the evidence that the $\tau_{\mathrm{HB}}$ index is a robust diagnostic to identify second parameter GGCs. It is worth mentioning the presence of four GGCs (NGC 6171, NGC 6362, NGC 6535, and NGC 7089) that are $\sim 2.5 \sigma$ away from the linear fit, but their position might also be affected by uncertainties in the absolute cluster age (see the horizontal error bar plotted in the bottom right corner).

To further investigate the impact of possible systematics on the cluster ages, we used the results by Salaris \& Weiss (2002) employing the Carretta \& Gratton (1997) metallicity scale, for their 43 GGCs in common with our sample. They divided the sample into four different metallicity bins and for each of them selected a calibrating cluster. They then estimated the absolute age of the calibrating cluster by using the vertical method, that is, the difference in visual magnitude $(\Delta V)$ between the HB luminosity level and the MSTO. For all the other clusters in a specific metallicity bin, they estimated the relative age with respect to the calibrating cluster by using the horizontal method, that is, the difference in colour $(\Delta(V-I)$ or $\Delta(B-V))$ between the main sequence TO and the base of the RGB.

Data are shown in the bottom panel of Fig. 18. Once again, we found that the $\tau_{\mathrm{HB}}$ index correlates with the cluster age. We performed the same linear fit as for the age estimates by VandenBerg et al. (2013), Leaman et al. (2013), and the coefficients are listed in Table 4 together with the standard deviation, which is equal to 1.89 . The red and the blue lines display the linear fit and the $1 \sigma$ limits, respectively. The difference between the second parameter clusters and the linear fit is larger than $2 \sigma$. This means that their peculiarity is independent of the adopted absolute age. Moreover, this plot also shows a few GGCs, roughly $2 \sigma$ away from the linear fit, already identified in the top panels: NGC 6171 and NGC 6535, plus a new one, NGC 6652.

We analysed the age and the metallicity dependencies of $\tau_{\mathrm{HB}}$, since they are considered as the main culprits affecting the HB morphology. However, the current findings further support the need for at least one more parameter to explain the observed variation in HB morphology. We assumed as a working hypothesis that the dispersion in the $\tau_{\mathrm{HB}}$-age diagram was caused by both age and helium variations (D'Antona et al. 2002; Caloi \& D'Antona 2005). Therefore, we decided to empirically remove the age dependence of $\tau_{\mathrm{HB}}$, and check whether the residuals in the reduced $\tau_{\mathrm{HB}}-[\mathrm{Fe} / \mathrm{H}]$ diagram correlate with the spread in helium of the cluster. This empirical "reduction" of $\tau_{\mathrm{HB}}$ to a single age accounts automatically for possible age-metallicity relations in the cluster sample, as well as for the dependence of $\tau_{\mathrm{HB}}$ on age at fixed metallicity. To perform this experiment we 


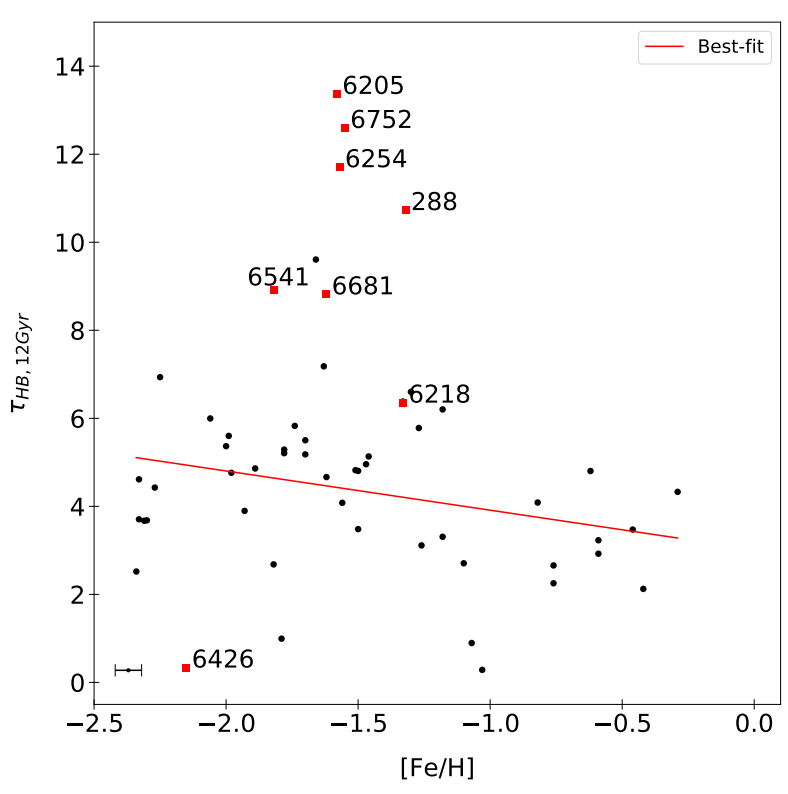

Fig. 19. $[\mathrm{Fe} / \mathrm{H}]$ vs. $\tau_{\mathrm{HB}, 12 \mathrm{Gyr}}$. The red line identifies the linear best-fit function of the plane, and red squares mark the eight second parameter clusters (see text for details).

reduced the individual $\tau_{\mathrm{HB}}$ measurements (Table 2 ) to the values they would have for an age of $12 \mathrm{Gyr}\left(\tau_{\mathrm{HB}, 12 \mathrm{Gyr}}\right.$, using the ages by VandenBerg et al. 2013), as detailed below.

We first calculated the index value at an age of $12 \mathrm{Gyr}$ as provided by the best-fit relation in the upper panel of Fig. 18 (value equal to 4.63); then, for each cluster, we calculated the values determined from the same best-fit relation but for the individual cluster ages. For each GGC we then calculated the difference $d \tau$ between the value expected at the cluster age and the value expected at $12 \mathrm{Gyr}$, and finally determined $\tau_{\mathrm{HB}, 12 \mathrm{Gyr}}=\tau_{\mathrm{HB}}-d \tau$.

Figure 19 shows the $\tau_{\mathrm{HB}, 12 \mathrm{Gyr}}$ values as a function of $[\mathrm{Fe} / \mathrm{H}]$. In contrast to what we found in Fig. 16, after eliminating on average the effect of age, we now have a linear best-fit relation between $\tau_{\mathrm{HB}, 12 \text { Gyr }}$ and the metal content (the red line in Fig. 19):

$\tau_{\mathrm{HB}, 12 \mathrm{Gyr}}=3.02-0.89 \cdot[\mathrm{Fe} / \mathrm{H}]$,

with dispersion $\sigma=1.64$. The red squares identify the second parameter clusters: they still attain $\tau_{\mathrm{HB}, 12 \mathrm{Gyr}}$ values far from the best-fit relation.

In a very recent paper, Milone et al. (2018) estimated the spread in initial helium content $(0.245 \leq Y \leq 0.4)$ for a sizeable sample of GGCs (57). They used data from the HST UV survey of Galactic GCs $(F 275 W, F 336 W$, and $F 438 W$ filters of the ultraviolet and visual channel of HST/WFC3 (UVIS/WFC3), Piotto et al. 2015; Milone et al. 2017) and from the Wide Field Channel of the Advanced Camera for Surveys (WFC/ACS) ( $F 606 W$ and $F 814 W$ photometry, Sarajedini et al. 2007; Dotter et al. 2011) programmes. The He spread values, $\partial Y_{\max }$, for each of the 56 GGCs in common with Milone et al. (2018) are listed in Col. 9 of Table 2.

The spread in helium content ranges from almost zero for NGC 6362, NGC 6535, and NGC 6717, up to $\sim 0.08$ for NGC 6388, NGC 6441, and NGC 7078. To further constrain the sensitivity of the new HB morphology index, we correlated the residuals of $\tau_{\mathrm{HB}, 12 \mathrm{Gyr}}$ of the best-fit function in Fig. 19 as a function of the metallicity, shown in Fig. 20.

The figure shows that the $\tau_{\mathrm{HB}, 12 \mathrm{Gyr}}$ index does not seem to be correlated with the spread in helium content. This means

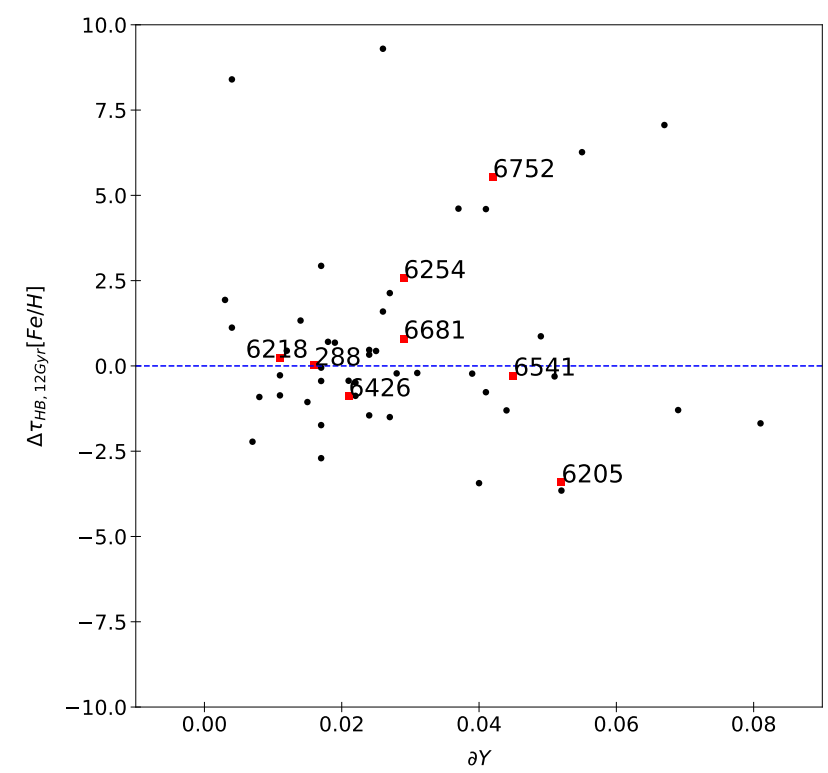

Fig. 20. Residuals of the corrected $\tau_{\mathrm{HB}, 12 \mathrm{Gyr}}$ as a function of the metal content (Carretta et al. 2009) versus the spread in helium content $\partial Y$ estimated by Milone et al. (2018). Red squares identify the second parameter globulars.

that, despite our attempt to limit the age and metallicity effects, the spread in helium content is not able to justify the observed spread in $\tau_{\mathrm{HB}}$. We note that we also estimated the residuals of the $\tau_{\mathrm{HB}, 12 \mathrm{Gyr}}$ using just space data (see Sect. 6) and related them to $\partial Y$ values, since the latter quantities are evaluated from the same HST data. Once again, despite the data homogeneity, we did not find any clear correlation with $\partial Y$ values.

\section{Comparison with synthetic horizontal branch models}

To constrain on a more quantitative basis the impact that both cluster age and spread in helium content have on the observed spread of the new HB morphology index, we decided to use a novel set of synthetic horizontal branch (SHB) models. The synthetic HB models have been computed employing HB tracks and progenitor isochrones from the $\alpha$-enhanced a Bag of Stellar Tracks and Isochrones (BaSTI) stellar model library (Pietrinferni et al. 2006) $)^{5}$ and a code fully described in Dalessandro et al. (2013). We considered three different metallicities, namely $[\mathrm{Fe} / \mathrm{H}]=-0.7$, $[\mathrm{Fe} / \mathrm{H}]=-1.62$, and $[\mathrm{Fe} / \mathrm{H}]=-2.14$ (all with $[\alpha / \mathrm{Fe}]=0.4$ ). The initial He abundances of the HB progenitors at these three metallicities are equal to $Y=0.245,0.246$, and 0.256 , respectively.

For each $[\mathrm{Fe} / \mathrm{H}]$ we calculated first a set of synthetic HBs for an age equal to $12 \mathrm{Gyr}$ (keeping $Y$ constant for each $[\mathrm{Fe} / \mathrm{H}]$ ), assuming the RGB progenitor loses an amount of mass $\Delta M=$ $0.28 M_{\odot}, \Delta M=0.21 M_{\odot}$, and $\Delta M=0.16 M_{\odot}$ for $\left.\mathrm{Fe} / \mathrm{H}\right]=-0.7$, $[\mathrm{Fe} / \mathrm{H}]=-1.62$, and $[\mathrm{Fe} / \mathrm{H}]=-2.14$, respectively. The mass loss is estimated with a $1 \sigma$ Gaussian spread equal to $0.01 M_{\odot}$, irrespective of the chemical composition. In addition, assuming the same age and RGB mass loss, we calculated SHBs for each metallicity with an uniform distribution of initial $Y$ for the progenitors, with a range $\partial Y=0.03$.

In brief, the synthetic HB code first draws randomly a value of $Y$ with a uniform probability distribution between $Y$ and

5 http://www.oa-teramo.inaf.it/BASTI 


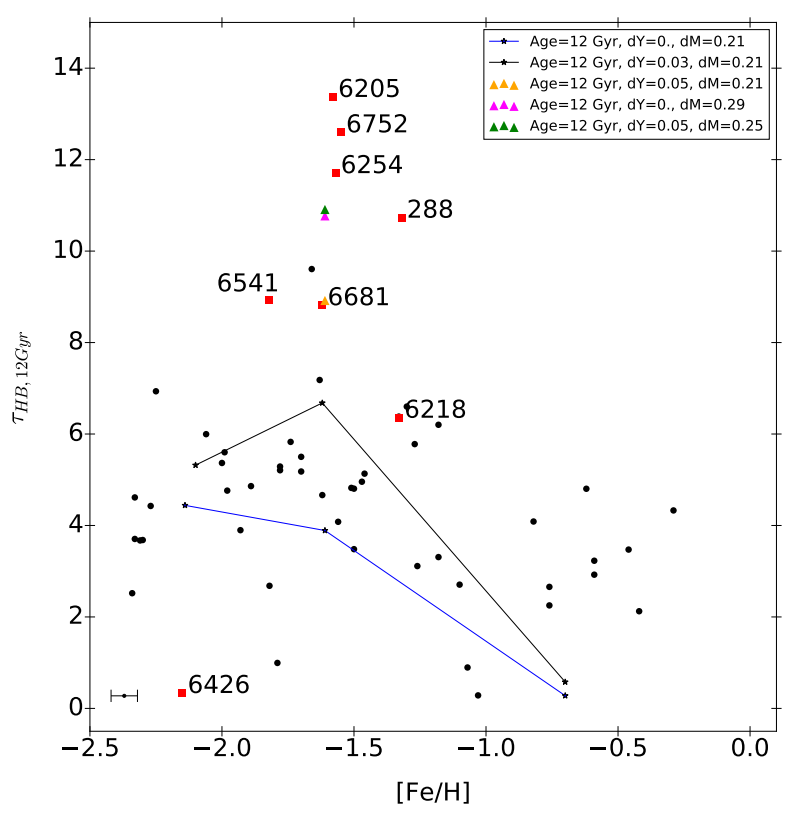

Fig. 21. New HB morphology index, $\tau_{\mathrm{HB}, 12 \mathrm{Gyr}}$, as a function of cluster iron abundance (Carretta et al. 2009). The two lines display synthetic $\mathrm{HB}$ models at fixed cluster age (12 Gyr), but either with a canonical helium content ( $\partial Y=0$, blue line) or with an internal spread in $\mathrm{He}$ of $\partial Y=0.03$ (black line). The coloured triangles identify three models for $[\mathrm{Fe} / \mathrm{H}]=-1.6$ for three different combinations of $\partial Y$ and $\Delta M$ (see legend). Red squares mark the eight second parameter clusters. The error bar on the left corner gives the 0.1 dex error on the metal content.

$Y+\partial Y(\partial Y=0$ for the models at constant $\mathrm{He})$ and determines the initial mass of the star at the RGB tip $\left(M_{\mathrm{TRGB}}\right)$ from interpolation amongst the BaSTI isochrones of the chosen age. The mass of the corresponding object evolving along the $\mathrm{HB}\left(M_{\mathrm{HB}}\right)$ is then calculated as $M_{\mathrm{HB}}=M_{\mathrm{TRGB}}-\Delta M$, where $\Delta M$ is drawn randomly according to a Gaussian distribution with the specified mean values and $\sigma$. The magnitudes of the synthetic star are then determined according to its position along the HB track with appropriate mass and $Y$ obtained by interpolation among the available set of HB tracks, after an evolutionary time $t$ has been randomly extracted. The value of $t$ is determined assuming that stars reach the ZAHB at a constant rate, employing a flat probability distribution ranging from zero to $t_{\mathrm{HB}}$, where $t_{\mathrm{HB}}$ is the time spent from the ZAHB to the He-burning shell ignition along the early asymptotic giant branch. The value of $t_{\mathrm{HB}}$ is set by the $\mathrm{HB}$ mass with the longest lifetime (the lowest masses for a given chemical composition). This implies that for some objects the randomly selected value of $t$ will be longer than its $t_{\mathrm{HB}}$, meaning that they have already evolved to the next evolutionary stages.

Figure 21 shows the comparison between the $\tau_{\mathrm{HB}, 12 \text { Gyr }}$ morphology index as a function of cluster iron abundance, and synthetic horizontal branch models. The blue line shows the SHB model at constant helium content $(\partial Y=0)$, while the black one is the synthetic model constructed assuming an internal spread in He content of $\partial Y=0.03$. This value was adopted according to the recent estimates provided by Milone et al. (2018).

In the metal-rich regime $([\mathrm{Fe} / \mathrm{H}] \geq-0.7)$ the predicted $\tau_{\mathrm{HB}}$ weakly changes with the spread in helium content. This is a consequence of the fact that SHB models predict an extreme red HB morphology, minimally affected by the intrinsic parameters we are taking into account. In the metal-intermediate regime $([\mathrm{Fe} / \mathrm{H}]=-1.62)$ the $\tau_{\mathrm{HB}}$ value predicted assuming an internal spread $\partial Y$ is characterized by a spike. This is due to the fact that at this metallicity values, a small mass variation causes significant changes in the SHB colour.

To explain the existence of our second parameter clusters, the triangles in Fig. 21 identify a further three different synthetic models for $[\mathrm{Fe} / \mathrm{H}]=-1.6$, the mean metallicity value of our second parameter clusters, at the fixed age of 12 Gyr. The yellow triangle shows the synthetic $\tau_{\mathrm{HB}}$ considering a higher spread in He compared to the one estimated by Milone et al. (2018) $(\partial Y=0.05)$ at the mass loss value we used for the blue and black models $(\Delta M=0.21)$; the magenta triangle identifies the model for $\partial Y=0$ and a mass loss of $\Delta M=0.29$ (higher of $0.08 M_{\odot}$ than the standard one); finally the green triangle identifies the synthetic $\tau_{\mathrm{HB}}$ value for the case with $\partial Y=0.05$ and $\Delta M=0.25$.

Therefore, Fig. 21 shows that if we want to model the estimated values of $\tau_{\mathrm{HB}, 12 \mathrm{Gyr}}$ we have three different possibilities. If we adopt the spread in He content estimated by Milone et al. (2018) (e.g. $\partial Y=0.05$ for NGC 6205, $\partial Y=0.04$ for NGC 6752, $\partial Y=0.03$ for NGC 6254, $\partial Y=0.03$ for NGC 6681, considering the second parameter cluster with $[\mathrm{Fe} / \mathrm{H}] \sim-1.6)$, we need to increase the mass loss value to 0.29 , which is a value greater by $0.08 M_{\odot}$ than the one able to fit the bulk of the clusters. If we fix the mass loss to the one needed to model the bulk of the clusters $(\Delta M=0.21)$, then we need to increase the spread in He content (from $\partial Y=0.03$ to, at least, $\partial Y=0.05$ ). Finally, we can explain the existence of the second parameter cluster also considering a higher mass loss together with a higher spread in He. We note that the adoption of a higher mass loss cannot be attributed to systematic errors in age estimations, since they should be on the order of 2-3 Gyr.

\section{Conclusions}

We took advantage of a sample of 64 GGCs for which we have homogeneous and accurate $U B V R I$ ground photometry and $V$ $(F 606 W), I(F 814 W)$ ACS/HST data (Sarajedini et al. 2007; Dotter et al. 2011), to introduce a new HB morphology index, named $\tau_{\mathrm{HB}}$, to investigate on a more quantitative basis the variation of the HB morphology when moving from the metal-poor to the metal-rich regime. We define $\tau_{\mathrm{HB}}$ as the ratio of the area below the cumulative number distribution in apparent magnitude $\left(A_{\mathrm{CND}}(I)\right)$ and in colour $\left(A_{\mathrm{CND}}(V-I)\right)$ of the entire HB region.

Even though the estimate of the $\tau_{\mathrm{HB}}$ index appears to be more complicated compared with HB morphology indices based either on star counts (HBR', Lee et al. 1990) or on specific evolutionary features (L1, L2, Milone et al. 2014), it offers several advantages. Indeed, we found that $\tau_{\mathrm{HB}}$ is a factor of seven more sensitive than the classical HBR' index and more than one order of magnitude more sensitive compared to L1 or L2 indices. Moreover, and even more importantly, the $\tau_{\mathrm{HB}}$ index shows a linear trend over the entire metallicity range $(-2.35 \leq[\mathrm{Fe} / \mathrm{H}] \leq-0.12)$ covered by GGCs. Furthermore, the $\tau_{\mathrm{HB}}$ index traces the HB luminosity function and it is independent of uncertainties affecting either the definition of different sub-groups (blue, red, variables) or the position of specific evolutionary features (L1, L2).

Moreover, to analyse the possible sensitivity of the HB morphology indices to the different contribution of inner and outer populations in GGCs, we estimated $\mathrm{HBR}^{\prime}$ and $\tau_{\mathrm{HB}}$ considering just space-based and just ground-based data. Comparing them to the results obtained using the combined HST and groundbased observations, we found that $\mathrm{HBR}^{\prime}$ has on average lower differences $(\sim 1.6 \%$, just ground-based, and $\sim 1.3 \%$, just spacebased) than the ones attained by $\tau_{\mathrm{HB}}(\sim 3.6 \%$, just ground-based, and $\sim 2.9 \%$, just space-based). For HBR' we observed major 
differences for the globulars in which the contribution of the red HB stars is mainly driven by HST observations. In $\tau_{\mathrm{HB}}$ analysis we found higher differences for small and concentrated clusters, or for those clusters which are larger but located at higher distances. In all these cases most of the cluster stars are located inside the ACS FoV and so the space-based data give the higher contribution to the global $\tau_{\mathrm{HB}}$. In general our data are selfconsistent and reliable and $\tau_{\mathrm{HB}}$ higher relative differences could mean that it is sensitive to the different contribution given by inner and outer populations observed in GGCs.

To quantify the sensitivity of the $\tau_{\mathrm{HB}}$ index on intrinsic stellar parameters, we investigated its dependence on cluster global properties (metallicity, absolute age, spread in He content). The main results of our analysis are the following:

Anti-correlation with cluster metallicity. We found a quadratic anti-correlation between $\tau_{\mathrm{HB}}$ and $[\mathrm{Fe} / \mathrm{H}]$. The majority of the metal-poor globulars $([\mathrm{Fe} / \mathrm{H}] \leq-1.5)$ have $\tau_{\mathrm{HB}}$ between $\sim 4$ and $\sim 9$, while the metal-intermediate ones $(-1.5 \leq[\mathrm{Fe} / \mathrm{H}] \leq-1.0)$ have values between $\sim 2$ and $\sim 5$. On the other hand, the metalrich clusters, with $[\mathrm{Fe} / \mathrm{H}] \geq-1.0$, attain $\tau_{\mathrm{HB}}$ values smaller than two.

Identification of second parameter clusters. We found a subsample of eight GGCs in the metal-poor and metalintermediate regime $(-1.82 \leq[\mathrm{Fe} / \mathrm{H}] \leq-1.32)$ which, at fixed metallicity, are characterized by $\tau_{\mathrm{HB}}$ values that are, on average, at least a factor of two larger than canonical clusters. The outlier clusters do not display any peculiarity in the $\mathrm{HBR}^{\prime}$ metallicity plane. To investigate their HB morphology, we selected three of them that sample the metal-rich $([\mathrm{Fe} / \mathrm{H}]=-1.33$, NGC 6218), metal-intermediate $([\mathrm{Fe} / \mathrm{H}]=-1.57$, NGC 6254), and metal-poor $([\mathrm{Fe} / \mathrm{H}]=-1.82, \mathrm{NGC} 6541)$ regimes. We compared their $I, V-I$ CMDs to those of three "regular" clusters with similar metallicities within the errors (NGC 362, NGC 6934, and NGC 6144). For each cluster pair, we find similar HBR' values but different $\tau_{\mathrm{HB}}$ values, with differences even on the order of three. For these reasons we can associate these clusters in our sample with the so-called second parameter clusters.

Correlation with cluster age. We investigate the relation between our HB morphology index $\tau_{\mathrm{HB}}$ and the absolute cluster age. To exclude possible dependence on the particular age estimation, we used the different homogeneous evaluations from VandenBerg et al. (2013), Leaman et al. (2013), Salaris \& Weiss (2002), finding a linear correlation between $\tau_{\mathrm{HB}}$ and the absolute age. We found that, in general, when moving from red to blue HB morphology, the GGCs become older. The second parameter clusters selected according to the $\tau_{\mathrm{HB}}$-metallicity plane appear to be peculiar also in the $\tau_{\mathrm{HB}}$-absolute age plane. In particular they attain cluster ages ranging from $\sim 11.5$ to $\sim 13 \mathrm{Gyr}$. Moreover, they seem to be characterized by bluer HB morphologies than the typical clusters.

Reductio ad unum. We limited the age impact on our analysis by reducing the $\tau_{\mathrm{HB}}$ values to the ones they would attain for an age of $12 \mathrm{Gyr}$. In contrast to what we originally found, we found a linear correlation between the corrected $\tau_{\mathrm{HB}, 12 \mathrm{Gyr}}$ values and $[\mathrm{Fe} / \mathrm{H}]$. However, the second parameter clusters are still located far from the best-fit linear relation of the plane.

Comparison with spread in helium content. We investigated our new HB morphology index in the context of internal helium content variation, supposed to be one of the main drivers of the HB morphology. We compared the residuals of the corrected $\tau_{\mathrm{HB}, 12 \mathrm{Gyr}}$ as a function of cluster metallicity with the internal spread in helium content, $\partial Y$, estimated by Milone et al. (2018), but we did not find a solid correlation.

Comparison with theory. We calculated a novel set of synthetic horizontal branch models to investigate the impact on $\tau_{\mathrm{HB}}$ of the spread in Helium content and mass loss along the branch, at the fixed age of $12 \mathrm{Gyr}$. We found that we can fit the second parameter clusters in our sample if: we fix the spread in He content to the one estimated by Milone et al. (2018) and we increase the mass loss value from $\Delta M=0.21$ (the one able to fit the bulk of the clusters) to $\Delta M=0.29$; we fix the mass loss to $\Delta M=0.21$ and we increase the spread in He content from $\partial Y=0.03$ (Milone et al. 2018) to, at least, $\partial Y=0.05$; we consider a higher mass loss together with an higher spread in $\mathrm{He}$ content.

Nature versus nurture. It is not clear whether the outlier clusters display a bluer HB morphology because they are intrinsically different (nature) or because the HB morphology is tracing a specific dynamical status of the cluster (nurture).

Acknowledgements. This work has made use of data from An ACS Survey of Galactic Globular Clusters programme in the context of the Hubble Space Telescope Treasury project. This investigation was partially supported by PRIN-INAF 2016 ACDC (P.I.:P. Caraveo). M.M. was supported by the Spanish Ministry of Economy and Competitiveness (MINECO) under the grant AYA2017-89076-P.

\section{References}

Anderson, J., Sarajedini, A., Bedin, L. R., et al. 2008, AJ, 135, 2055 Bono, G., \& Stellingwerf, R. F. 1994, ApJS, 93, 233

Bono, G., Marconi, M., \& Stellingwerf, R. F. 1999, ApJS, 122, 167

Braga, V. F., Stetson, P. B., Bono, G., et al. 2016, AJ, 152, 170

Brown, T. M., Sweigart, A. V., Lanz, T., et al. 2010, ApJ, 718, 1332

Buonanno, R. 1993, in The Globular Cluster-Galaxy Connection, eds. G. H. Smith, \& J. P. Brodie, ASP Conf. Ser., 48, 131

Calamida, A., Strampelli, G., Rest, A., et al. 2017, AJ, 153, 175

Caloi, V., \& D’Antona, F. 2005, A\&A, 435, 987

Carretta, E., \& Gratton, R. G. 1997, A\&AS, 121, 95

Carretta, E., Bragaglia, A., Gratton, R., D’Orazi, V., \& Lucatello, S. 2009, A\&A, 508,695

Carretta, E., Bragaglia, A., Gratton, R. G., et al. 2014, A\&A, 564, A60

Castellani, M., Caputo, F., \& Castellani, V. 2003, A\&A, 410, 871

Chaboyer, B., McArthur, B. E., O’Malley, E., et al. 2017, ApJ, 835, 152

Clement, C. M., Muzzin, A., Dufton, Q., et al. 2001, AJ, 122, 2587

Dalessandro, E., Salaris, M., Ferraro, F. R., Mucciarelli, A., \& Cassisi, S. 2013, MNRAS, 430, 459

D’Antona, F., Caloi, V., Montalbán, J., Ventura, P., \& Gratton, R. 2002, A\&A, 395,69

Di Cecco, A., Bono, G., Prada Moroni, P. G., et al. 2015, AJ, 150, 51

D’Orazi, V., Magurno, D., Bono, G., et al. 2018, ApJ, 855, L9

Dotter, A., Chaboyer, B., Jevremović, D., et al. 2007, AJ, 134, 376

Dotter, A., Sarajedini, A., \& Anderson, J. 2011, ApJ, 738, 74

Dutra, C. M., \& Bica, E. 2000, A\&A, 359, 347

Freeman, K. C., \& Norris, J. 1981, ARA\&A, 19, 319

Gratton, R. G., Carretta, E., Bragaglia, A., Lucatello, S., \& D’Orazi, V. 2010, A\&A, 517, A81

Harris, W. E. 1996, AJ, 112, 1487

Iannicola, G., Monelli, M., Bono, G., et al. 2009, ApJ, 696, L120

Kraft, R. P., \& Ivans, I. I. 2003, PASP, 115, 143

Lanzoni, B., Ferraro, F. R., Mucciarelli, A., et al. 2018, ApJ, 861, 16

Leaman, R., VandenBerg, D. A., \& Mendel, J. T. 2013, MNRAS, 436, 122

Lee, Y. W. 1989, PhD Thesis, Yale University

Lee, Y.-W., Demarque, P., \& Zinn, R. 1990, ApJ, 350, 155

Lee, Y.-W., Demarque, P., \& Zinn, R. 1994, ApJ, 423, 248

Marconi, M., Coppola, G., Bono, G., et al. 2015, ApJ, 808, 50

Milone, A. P., Marino, A. F., Dotter, A., et al. 2014, ApJ, 785, 21

Milone, A. P., Piotto, G., Renzini, A., et al. 2017, MNRAS, 464, 3636

Milone, A. P., Marino, A. F., Renzini, A., et al. 2018, MNRAS, 481, 5098

Monelli, M., Testa, V., Bono, G., et al. 2015, ApJ, 812, 25

Pancino, E., Galfo, A., Ferraro, F. R., \& Bellazzini, M. 2007, ApJ, 661, L155

Pietrinferni, A., Cassisi, S., Salaris, M., \& Castelli, F. 2006, ApJ, 642, 797 
Piotto, G., Milone, A. P., Bedin, L. R., et al. 2015, AJ, 149, 91

Pritzl, B. J., Smith, H. A., Stetson, P. B., et al. 2003, AJ, 126, 1381

Recio-Blanco, A., Aparicio, A., Piotto, G., de Angeli, F., \& Djorgovski, S. G. 2006, A\&A, 452, 875

Richer, H. B., Goldsbury, R., Heyl, J., et al. 2013, ApJ, 778, 104

Salaris, M., \& Weiss, A. 2002, A\&A, 388, 492

Salaris, M., \& Cassisi, S. 2005, Evolution of Stars and Stellar Populations (Wiley)

Sandage, A. R. 1953, AJ, 58, 61

Sandage, A., \& Wildey, R. 1967, ApJ, 150, 469

Sarajedini, A., Bedin, L. R., Chaboyer, B., et al. 2007, AJ, 133, 1658

Searle, L., \& Zinn, R. 1978, ApJ, 225, 357

Stetson, P. B., Catelan, M., \& Smith, H. A. 2005, PASP, 117, 1325

Stetson, P. B., Braga, V. F., Dall'Ora, M., et al. 2014, PASP, 126, 521

Stetson, P. B., Pancino, E., Zocchi, A., Sanna, N., \& Monelli, M. 2019, MNRAS, 485,3042

van den Bergh, S. 1967, AJ, 72, 70

VandenBerg, D. A., Brogaard, K., Leaman, R., \& Casagrande, L. 2013, ApJ, 775, 134

Villanova, S., Geisler, D., Piotto, G., \& Gratton, R. G. 2012, ApJ, 748, 62

Weiss, A., Schlattl, H., Salaris, M., \& Cassisi, S. 2004, A\&A, 422, 217

Zinn, R., \& West, M. J. 1984, ApJS, 55, 45

Zoccali, M., Cassisi, S., Bono, G., et al. 2000, ApJ, 538, 289

Zocchi, A., Gieles, M., \& Hénault-Brunet, V. 2017, MNRAS, 468, 4429

\section{Appendix A: Peculiar globular clusters}

The classical HB morphology index (HBR') estimations for NGC 6304, NGC 6426, NGC 6624, Lynga 7, and Palomar 2 and the $\tau_{\mathrm{HB}}$ indices for NGC 6426, NGC 6624, and NGC 6652 were derived using $I, V$-bands from ACS-HST because we lack ground-based catalogues. For these globulars we considered all the stars in the HST field and within the tidal radius in groundbased telescope fields.

Due to the high field contamination in ground observations, for the clusters NGC 6388 and NGC 6441, we used only ACS data. Despite this, the sample is statistically good enough to analyse their HB morphology, even if the ACS FoV does not cover the entire extent of these two clusters.

Owing to the low number of HB stars in Lynga 7 and NGC 6304, we obtain $\tau_{\mathrm{HB}}=0$ and so, these clusters have not been included in the analysis of our new index. Finally, we note that ground-based data for NGC 104, NGC 5272, NGC 5466, NGC 5927, NGC 6362, NGC 6397, and NGC 6752 do not reach the tidal radius.

\section{Appendix B: Notes on individual outliers}

In the following we give notes about the clusters we can define as outliers either in the $\tau_{\mathrm{HB}}-[\mathrm{Fe} / \mathrm{H}]$ plane or in the $\tau_{\mathrm{HB}}$-age plane or both.

NGC 6218. Outlier of $\tau_{\mathrm{HB}}-[\mathrm{Fe} / \mathrm{H}]$, while we cannot consider it an outlier in $\tau_{\mathrm{HB}}$-age planes (it is located under the $2.0 \sigma$ levels). Both ground-based and space data are of good quality. It has been identified as a second parameter cluster.

NGC 288. Outlier of $\tau_{\mathrm{HB}}-[\mathrm{Fe} / \mathrm{H}]$ and of the $\tau_{\mathrm{HB}}$-age planes. Both ground-based and space data are of good quality. It has been identified as a second parameter cluster.

NGC 6205. Outlier of $\tau_{\mathrm{HB}}-[\mathrm{Fe} / \mathrm{H}]$ and of the $\tau_{\mathrm{HB}}$-age planes. Both ground-based and space data are of good quality. It has been identified as a second parameter cluster.

NGC 6254. Outlier of $\tau_{\mathrm{HB}}-[\mathrm{Fe} / \mathrm{H}]$ and of the $\tau_{\mathrm{HB}}$-age planes. Both ground-based and space data are of good quality. It has been identified as a second parameter cluster.

NGC 6426. Outlier of $\tau_{\mathrm{HB}}-[\mathrm{Fe} / \mathrm{H}]$ and of $\tau_{\mathrm{HB}}$-age planes. We have only space photometry. Among the second parameter clusters, it is the one attaining the lowest value in $\tau_{\mathrm{HB}}$.

NGC 6752. Outlier of $\tau_{\mathrm{HB}}-[\mathrm{Fe} / \mathrm{H}]$ and of the $\tau_{\mathrm{HB}}$-age planes. Both ground-based and space data are of good quality, but ground data do not reach the globular tidal radius. It has been identified as a second parameter cluster.

NGC 6541. Outlier of $\tau_{\mathrm{HB}}-[\mathrm{Fe} / \mathrm{H}]$ and of the VandenBerg et al. (2013), Leaman et al. (2013) $\tau_{\mathrm{HB}}$-age plane. We have no age estimation from Salaris \& Weiss (2002). Both ground-based and space data are of good quality. It has been identified as a second parameter cluster.

NGC 6681. Outlier of $\tau_{\mathrm{HB}}-[\mathrm{Fe} / \mathrm{H}]$ and of the $\tau_{\mathrm{HB}}$-age planes. Both ground-based and space data are of good quality. It has been identified as a second parameter cluster.

NGC 6362. Outlier of $\tau_{\mathrm{HB}}$-age plane for age estimations from VandenBerg et al. (2013), Leaman et al. (2013). Both ground-based and space data are of good quality.

NGC 6535. Outlier of $\tau_{\mathrm{HB}}$-age planes (above $2.5 \sigma$ limit in the VandenBerg et al. 2013; Leaman et al. 2013 plane and 2.0 $\sigma$ limit in the Salaris \& Weiss 2002 one). Both ground-based and space data are of good quality.

NGC 6652. Outlier of the Salaris \& Weiss (2002) $\tau_{\mathrm{HB}}$-age plane. Both ground-based and space data are of good quality.

NGC 7089. Outlier of the VandenBerg et al. (2013), Leaman et al. (2013) $\tau_{\mathrm{HB}}$-age plane. We have no age estimation from Salaris \& Weiss (2002). Both ground-based and space data are of good quality.

NGC 6171. Outlier in the $\tau_{\mathrm{HB}}$-age planes (above the $2.5 \sigma$ limit in VandenBerg et al. 2013 and the $2.0 \sigma$ limit in Salaris $\&$ Weiss 2002). Both ground-based and space data are of good quality. 